\title{
Vaccine adjuvants CpG (oligodeoxynucleotides ODNs), MPL (3-O- deacylated monophosphoryl lipid A) and naloxone-enhanced Th1 immune response to the Plasmodium vivax recombinant thrombospondin-related adhesive protein (TRAP) in mice
}

\author{
Saeed Nazeri ${ }^{1} \cdot$ Sedigheh Zakeri ${ }^{1}$ (D) Akram A. Mehrizi ${ }^{1} \cdot$ Navid D. Djadid $^{1} \cdot$ Georges Snounou $^{2} \cdot$ Chiara Andolina $^{3}$. \\ François Nosten ${ }^{3,4}$
}

Received: 20 August 2017 / Accepted: 23 May 2018 / Published online: 9 June 2018

(c) Springer-Verlag GmbH Germany, part of Springer Nature 2018

\begin{abstract}
Despite considerable efforts toward vaccine development over decades, there is no available effective vaccine against Plasmodium vivax. Thrombospondin-related adhesive protein of P. vivax (PvTRAP) is essential for sporozoite motility and invasions into mosquito's salivary gland and vertebrate's hepatocyte; hence, it is a promising target for pre-erythrocytic vaccine. In the current investigation, the role of antibodies and cellular immune responses induced by purified recombinant PvTRAP (rPvTRAP) delivered in three adjuvants, naloxone (NLX), CpG oligodeoxynucleotides ODN1826 (CpG-ODN), and 3-O-deacylated monophosphoryl lipid A (MPL), alone and in combination was evaluated in immunized C57BL/6 mice. The highest level and the avidity of anti-PvTRAP IgG (mean OD $\left.{ }_{490 \mathrm{~nm}} 2.55\right), \mathrm{IgG}_{2} \mathrm{~b}\left(\right.$ mean OD $\left._{490 \mathrm{~nm}} 1.68\right)$, and IgG2c (mean $\mathrm{OD}_{490 \mathrm{~nm}}$ 1.466) were identified in the group received rPvTRA/NLX-MPL-CpG. This group also presented the highest IgG2c/ IgG1 (2.58) and $\operatorname{IgG} 2 \mathrm{~b} / \mathrm{IgG} 1$ (2.95) ratio when compared to all other groups, and among the adjuvant groups, the lowest $\mathrm{IgG} 2 \mathrm{c} / \mathrm{IgG1}(1.86)$ and $\mathrm{IgG} 2 \mathrm{~b} / \mathrm{IgG} 1$ (2.25) ratio was observed in mice receiving rPvTRAP/NLX. Mice receiving rPvTRAP/ adjuvants induced significantly the higher levels of interferon gamma (IFN- $\gamma$ ), low level of detectable IL-10, and no detectable IL-4 production. The present result revealed that PvTRAP is immunogenic and its administration with CPG, MPL, and NLX in C57BL/6 mice induced Th1 immune response. Besides, the rPvTRAP delivery in the mixed formulation of those adjuvants had more potential to increase the level, avidity, and persistence of anti-TRAP antibodies. However, it warrants further assessment to test the blocking activity of the produced antibodies in immunized mice with different adjuvant formulations.
\end{abstract}

Keywords Malaria $\cdot$ Plasmodium vivax $\cdot$ Vaccine $\cdot$ TRAP $\cdot$ Adjuvant

\section{Introduction}

Electronic supplementary material The online version of this article (https://doi.org/10.1007/s00430-018-0545-2) contains supplementary material, which is available to authorized users.

Sedigheh Zakeri

zakeris@yahoo.com; zakeris@pasteur.ac.ir

1 Malaria and Vector Research Group (MVRG), Biotechnology Research Center (BRC), Pasteur Institute of Iran, Pasteur Avenue, Tehran, P.O. Box 1316943551, Iran

2 Sorbonne Universités, UPMC Univ Paris 06, Inserm (Institut National de la Santé et de la Recherche Medicale), Centre d'Immunologie et des Maladies Infectieuses (Cimi-Paris),
Plasmodium vivax is the major cause of malaria outside Sub-Saharan Africa and produces significantly less mortality than $P$. falciparum $[1,2]$, though increasing number of publications have reported severe diseases as a result of $P$.

UMR 1135, ERL CNRS 8255 (Centre National de la Recherche Scientifique), 91 Boulevard de l'Hôpital, 75013 Paris, France

3 Shoklo Malaria Research Unit, Mahidol-Oxford Tropical Medicine Research Unit, Faculty of Tropical Medicine, Mahidol University, Mae Sot, Thailand

4 Centre for Tropical Medicine and Global Health, Nuffield Department of Medicine, University of Oxford, Oxford, UK 
vivax infection [3-5]. Moreover, it has been indicated that the elimination of $P$. vivax rather than P. falciparum is often technically more challenging [6], due to the formation of dormant liver stages (hypnozoites) that reactivate several weeks to months after primary infection to cause symptomatic disease. Hence, there is no doubt that a vaccine is the most effective option for the control of vivax malaria not only to prevent the morbidity associated with the disease but also to prevent the potential spread of vivax malaria due to the reactivation of hypnozoites in currently non-endemic areas. However, the global efforts toward development of a vaccine against $P$. vivax are limited due to the absence of a continuous in vitro culture for $P$. vivax, which has a negative effect on obtaining the large amounts of parasites target antigens for whole vaccine development.

Experience has shown that developing vaccines against intracellular pathogens (such as Plasmodium), which require protective Th1 and cell mediate immunity, is more difficult than extracellular pathogens. In addition, in some cases, such as malaria vaccine development, due to the lack of continuous culture for most species of Plasmodium, the only solution could be subunit vaccines; therefore, additional immunostimulants, such as an efficient adjuvant [7-10], is highly important to induce a protective and long-lived immune response [11]. Adjuvant selection may be based on several parameters, including potential use in human, the physical and chemical natures of the vaccine antigen, type of desirable immune response, age of the target population, and the route of vaccine administration. In fact, the selection of an incorrect adjuvant may exhibit a specific vaccine antigen insufficient [12]. Thus, the selection of adjuvant system (AS) is critical to induce proper and protective immune responses, and to increase antibodies with greater avidity, with the potential for neutralization, and with the ability to modulate Th1 cell predominance [13, 14].

It is known that Th1-type antibodies (IgG1 and IgG3 in human and $\mathrm{IgG} 2 \mathrm{a} / \mathrm{c}$, and $\operatorname{IgG} 2 \mathrm{~b}$ in mice), cytokines [interferon gamma (IFN- $\gamma$ )], and $\mathrm{CD} 8+\mathrm{T}$ cells are important for the immune response against the malaria parasite [15]. One of the most significant issues that need to be considered in producing effective malaria subunit vaccines is having strong and effective adjuvants that help to elicit a proper and long-lasting immune response to maximize protection from disease. In this regard, the history of development of the RTS,S vaccine confirms the critical importance of appropriate adjuvants for the efficacy of vaccines based on purified recombinant antigens [16-21]. When the same malaria antigen that was used in RTS,S is formulated with a single adjuvant such as alum, 3-O-deacylated monophosphoryl lipid A (MPL) alone, and/or oil-in-water emulsion, the protective immune response failed [16], suggesting the importance of mixture of the adjuvants or ASs in the development of effective subunit malaria vaccine $[16,17,19-21]$. Therefore, AS series such as AS01 (liposomes containing MPL and QS21) [22], AS02 (an oil-in-water emulsion containing MPL and QS21) [23], AS04 (the combination of MPL and Alum) [24], and AS05 (liposomes containing aluminum hydroxide, MPL, and QS21) [17] seems to be more efficient than single-used adjuvants $[25,26]$. AS has also shown to have a critical role in the induction of potent, long-lasting antibody, and T-cell responses in diseases such as HPV [27], influenza [28], and meningococcus [29].

In the present study, three adjuvants, synthetic $\mathrm{CpG}$ oligodeoxynucleotides ODN1826 (CpG-ODNs), MPL, and naloxone (NLX), which are already in use or have the potential to be used in humans, are combined with the recombinant antigen as immunostimulants to induce potent Th1 response. The previous works have suggested that NLX, an opioid antagonist, has the ability to shift the immune response to a Th1 profile [30-36] via increasing the IFN- $\gamma$ level $[35,36]$. Thus, it appears that NLX has fundamental immune-modulating effects on the immune system, which is mediated directly through opioid receptors on immune cells and/or indirectly through the central nervous system [37]. Toll-like receptors (TLRs) are predominantly expressed by the first- line professional phagocytes such as neutrophils, macrophages, and DCs. Ten TLRs have been identified in humans for recognizing extracellular microbial structures as well as viral and bacterial nucleic acids [38]. MPL, as an immunomodulator, a TLR4 agonist, is a non-toxic derivative of the lipopolysaccharide (LPS) endotoxin expressed in the outer membrane of Salmonella minnesota. It induces a potent stimulation of the Th1 responses and is characterized by the secretion of pro-inflammatory cytokines and cytotoxic antibodies and also the activation of CTLs [39]. MPL, including adjuvant formulations (mainly ASO4, ASO2, and AS01), has been approved for human use and has currently been under clinical evaluation for several vaccines against cancer and other infectious diseases [40-43]. In addition, CpG-ODN is a potent Th1-polarizing adjuvant that activates antigen-presenting cells endosomally through TLR9. CpGODNs comprise short synthetic DNA molecules containing unmethylated $\mathrm{CpG}$ motifs (cytosine phosphoguanosine dinucleotides common in bacteria and virus) [44]. It possesses a high potential to induce innate immunity as well as specific humoral and Th1-cell-mediated responses [45]. For this reason, CpG-ODNs have also been postulated as adjuvants for cancer [46] and allergy [47] vaccines. Regarding malaria, CPG7909 has clinically been tested in combination with carrier adjuvants to increase and modulate the immune response.

Pre-erythrocytic vaccine strategies aim to generate an antibody response that is able to block sporozoite invasion of liver cells [48] and to induce opsonisation [49], as well as to elicit a cell-mediated immune response to interfere with the intra-hepatic multiplication cycle of the parasites, e.g., 
by killing the parasite-infected hepatocytes. Thrombospondin-related adhesive protein (TRAP) is a promising malaria vaccine candidate and is an antigen from the pre-erythrocyte stage of the malaria parasite Plasmodium. The protein has been found to be present in the sporozoite micronemes that appears to be released to the parasite surface, immediately before the invasion process, and its ectodomain region consists of an A domain, a TSR, and a repeat region. It is a transmembrane protein that is required for sporozoite gliding motility, and it has been found that this protein with circumsporozoite protein is essential for the process of malaria sporozoite infection of the hepatocyte $[50,51]$. These findings have important implications for the potential use of this antigen in a subunit vaccine against $P$. vivax parasite.

Therefore, in an attempt to improve the immunogenicity of a malaria subunit vaccine, the purified recombinant PvTRAP (rPvTRAP) delivered in three putative adjuvants, NLX, CpG-ODN, and MPL, alone and in combination that interact with different TLRs was evaluated in immunized C57BL/6 mice. This study was performed due to the requirement for protective immunity (particularly Th1 response) against the pre-erythrocyte stage of $P$. vivax parasite and also because of a need for an adjuvant or AS that induces strong and long-lasting Th1 response in vivax vaccine development. In this light, antibody-dependent immune mechanism (IgG, IgG1, IgG2b, IgG2c, and IgG3 responses) as well as the IFN- $\gamma$, IL-4, and IL-10 cytokines were determined in post-immunized mouse sera. Moreover, both induced humoral and cellular immunity responses were compared with complete Freund's adjuvant (CFA) as a well-known but not a human-compatible adjuvant.

\section{Materials and methods}

\section{Expression, purification, and confirmation of expressed rPvTRAP protein in $E$. coli host}

The ectodomain of PvTRAP representing amino acids 25-488 (GenBank Accession No. KT207838) [52] was cloned, expressed, and purified as described previously [53]. In brief, this part of PvTRAP was cloned into pET23a vector and expressed in Escherichia coli Rosetta (DE3), as a fusion protein with C-terminal hexa-His tag. The protein expression was induced by isopropyl-beta-D-thiogalactopyranoside (IPTG, Thermo Scientific, Waltham, Massachusetts, USA) with continuous shaking $(180 \mathrm{rpm})$ at $37^{\circ} \mathrm{C}$ for $4 \mathrm{~h}$. The fusion protein was purified with the Ni-NTA agarose (Qiagen, Hilden, Germany) and eluted with an elution buffer containing $200 \mathrm{mM}$ imidazole (pH 7.9). In the next step, it was desalted against $0.5 \times$ phosphate-buffered saline (PBS) using a Econo-Pac 10 DG columns (Bio-Rad, Hercules, CA, USA) according to the manual described by manufacturer and concentrated with a concentrator (Eppendorf, Hamburg, Germany). The concentration of proteins was measured by Bradford assay with a spectrophotometer (DeNovix, Wilmington, DE, USA) at $595 \mathrm{~nm}$. The desalted proteins were analyzed under reducing [with $1 \%$ sodium dodecyl sulfate (SDS) and 2\% 2ME] and non-reducing (with SDS and without 2ME) conditions by $10 \%$ SDS-polyacrylamide gel electrophoresis (SDS-PAGE). To confirm the purified recombinant proteins, Western blot assay was carried out by the standard protocols using anti-His antibody (Penta-His Antibody; Qiagen) and with P. vivax-infected human sera [53]. Finally, the level of bacterial endotoxin was determined using the LAL chromogenic kit (Lonza, Walkersville, MD, USA), in the Quality Control Unit of the Recombinant Protein Production Complex of Pasteur Institute of Iran (Karaj, Iran).

\section{Mice immunization with rPvTRAP}

Inbred female C57BL/6 mice were obtained from the Laboratory Animal Science Department, Pasteur Institute of Iran, (Karaj, Iran). The mice were housed under specific pathogen-free conditions in the animal care facility and allowed to adapt to housing conditions for 1 week prior to the experiment. All the experimental protocols were approved by the Committee of Animal Ethics of Pasteur Institute of Iran (IR. PII.REC.1395.36) and performed accordingly. 6-8-week-old female C57BL/6 mice were randomly divided into 12 groups ( $n=15$ in each group) and were immunized by subcutaneous injection with purified rPvTRAP ( $5 \mu \mathrm{g}$ at prime and $2.5 \mu \mathrm{g}$ at boost) alone or in combination with NLX, MPL, and $\mathrm{CpG}$ adjuvants at the base of tail, three times at 14-day intervals (Table 1). In negative controls, the mice were similarly immunized with sterile PBS $(0.5 \times \mathrm{PBS}, \mathrm{pH} 7.4)$, NLX ( $5 \mathrm{mg} / \mathrm{kg}$ body weight; Sigma-Aldrich Co., St. Louis, MO, USA), MPL ( $1 \mathrm{mg} / \mathrm{ml}$; InvivoGen, San Diego, CA, USA), CpG (2 mg/ml, InvivoGen, San Diego, CA, USA), and also with the combination of NLX $(0.1 \mathrm{mg} / \mathrm{kg})-\mathrm{MPL}(10 \mu \mathrm{g} /$ mouse)-CpG (5 $\mu \mathrm{g} /$ mouse) (Table 1$)$. In the adjuvant control groups, mice were immunized with rPvTRAP emulsified in CFA (for the first dose) or IFA (subsequent doses) (both form Sigma-Aldrich Co.,) with 1:1, volume/volume prior to the injection. For evaluation of the humoral responses to rPvTRAP and their persistence, sera samples were collected from the tail vein before immunization (as pre-immune sera) as well as 10, 24, 38, and 180 days after the first immunization (Table 1). All sera were stored at $-20{ }^{\circ} \mathrm{C}$ until use.

\section{Heparan sulfate-binding assay}

Heparan sulfate-binding assay was performed as described before [54] with some modifications. Briefly, Maxisorp flatbottom 96-well microplates (Jet Biofil, Guangzhou, China) 
Table 1 Mice immunization approach and bleeding

\begin{tabular}{|c|c|c|c|c|c|c|c|c|c|c|}
\hline \multicolumn{11}{|c|}{ Immunization and bleeding } \\
\hline \multirow[t]{2}{*}{ Groups } & \multicolumn{2}{|c|}{ Prime (day 0) } & & \multicolumn{2}{|c|}{ Boost 1 (day 14) } & & \multicolumn{2}{|c|}{ Boost 2 (day 28) } & & \\
\hline & Antigen & Adjuvant & & Antigen & Adjuvant & & Antigen & Adjuvant & & \\
\hline 1 & rPvTRAP & - & \multirow{12}{*}{$\begin{array}{c}\text { Bleeding } 1 \\
\text { (day 10) }\end{array}$} & rPvTRAP & - & Bleeding 2 & rPvTRAP & - & \multirow{12}{*}{$\begin{array}{c}\text { Bleeding } 3 \\
\quad \text { (day 38) }\end{array}$} & \multirow{12}{*}{$\begin{array}{r}\text { Bleeding } 4 \\
\quad(\text { day } 180)\end{array}$} \\
\hline 2 & rPvTRAP & CFA & & rPvTRAP & IFA & (day 24) & rPvTRAP & IFA & & \\
\hline 3 & rPvTRAP & NMC & & rPvTRAP & NMC & & rPvTRAP & NMC & & \\
\hline 4 & rPvTRAP & NLX & & rPvTRAP & NLX & & rPvTRAP & NLX & & \\
\hline 5 & rPvTRAP & MPL & & rPvTRAP & MPL & & rPvTRAP & MPL & & \\
\hline 6 & rPvTRAP & $\mathrm{CpG}$ & & rPvTRAP & $\mathrm{CpG}$ & & rPvTRAP & $\mathrm{CpG}$ & & \\
\hline $7(\mathrm{NC})$ & - & PBS & & - & PBS & & - & PBS & & \\
\hline $8(\mathrm{NC})$ & - & CFA & & - & IFA & & - & IFA & & \\
\hline $9(\mathrm{NC})$ & - & NMC & & - & NMC & & - & NMC & & \\
\hline $10(\mathrm{NC})$ & - & NLX & & - & NLX & & - & NLX & & \\
\hline $11(\mathrm{NC})$ & - & MPL & & - & MPL & & - & MPL & & \\
\hline $12(\mathrm{NC})$ & - & $\mathrm{CpG}$ & & - & $\mathrm{CpG}$ & & - & $\mathrm{CpG}$ & & \\
\hline
\end{tabular}

Mice received $200 \mu \mathrm{l} \operatorname{rPvTRAP}(5 \mu \mathrm{g}$ at prime and $2.5 \mu \mathrm{g}$ at boost) alone and in the presence of CFA, NLX $(0.1 \mathrm{mg} / \mathrm{mouse}), \mathrm{MPL}(10 \mu \mathrm{g} /$ mouse), CpG (5 $\mu \mathrm{g} / \mathrm{mouse})$, and NLX-MPL-CpG $(0.1 \mathrm{mg} / \mathrm{mouse}, 10 \mu \mathrm{g} / \mathrm{mouse}$, and $5 \mu \mathrm{g} / \mathrm{mouse}$, respectively) adjuvants subcutaneously at the base of tail. Control mice received PBS, CFA/IFA (as the reference adjuvant), NLX, CPG, MPL, and NLX-MPL-CpG

$C F A$ complete Freund's adjuvant, IFA incomplete Freund's adjuvant, $N L X$ naloxone, $M P L$ monophosphoryl lipid A, NMC NLX-MPL-CpG, NC negative control

were coated with $100 \mu \mathrm{l} /$ well of $10 \mu \mathrm{g} / \mathrm{ml}$ (diluted in water) of heparan sulfate from bovine kidney (Sigma-Aldrich Co.), and the control wells were incubated with $100 \mu \mathrm{l}$ of $10 \mu \mathrm{g}$ $\mathrm{BSA} / \mathrm{ml}$, and all plates were incubated at $37^{\circ} \mathrm{C}$ overnight. Next, the plates were washed with $0.05 \%$ Tween-20 (SigmaAldrich Co.) in PBS (PBS-T) and then were blocked with a $1 \%$ bovine serum albumin (BSA) in PBS at room temperature (RT) for an hour. After washing steps, the plates were incubated with the different concentrations of rPvTRAP antigen (starting at 60-0.625 $\mu \mathrm{g} / \mathrm{ml}$ ) in PBS at RT for $2 \mathrm{~h}$. After washing with PBS-T, the binding of rPvTRAP to heparan sulfate was detected by the addition of $100 \mu \mathrm{l}$ mouse polyclonal antibodies against rPvTRAP (obtained 38 days after the first immunization) for $2 \mathrm{~h}$, followed by goat anti-mouse IgG-horseradish peroxidase (HRP) conjugate (1:25,000; Sigma-Aldrich Co.) at RT for an hour. The reaction was developed using $o$-phenylenediamine/ $\mathrm{H}_{2} \mathrm{O}_{2}(\mathrm{OPD}$, Sigma-Aldrich Co.), as a substrate, and stopped with $2 \mathrm{~N}$ $\mathrm{H}_{2} \mathrm{SO}_{4}$. The optical density (OD) at $490 \mathrm{~nm}$ was read using a microplate reader (BioTek, Winooski, VT, USA).

\section{HepG2 liver cell-binding assay}

A hepatoma cell line, HepG2 (IBRC C10096; Iranian Biological Resource Center, Tehran, Iran), was maintained in Dulbecco Minimum Essential Medium (DMEM) Ham's F12 (Biowest, Nuaille, France) supplemented with $10 \%$ fetal calf serum (FCS, Sigma-Aldrich, Co.), 2 mM L-glutamine (Biowest), and 1\% penicillin-streptomycin (Sigma-Aldrich
Co.). The cells were removed from culture flasks with Trypsin-EDTA $(0.25 \%$ Trypsin, 1 mM EDTA, Gibco BRL, Life Technologies, Rockville, MS, USA) and pelleted at $3000 \times g$ for $15 \mathrm{~min}$. The cell pellet was resuspended in complete DMEM medium to a concentration of $10^{6}$ cells/ ml. Subsequently, $10^{5}$ cells/well were plated in 96-well cell culture plates (Orange Scientific, Braine-I'Alleud, Belgium) and allowed to grow in $5 \% \mathrm{CO}_{2}$ at $37{ }^{\circ} \mathrm{C}$ overnight. The cells were washed twice with the medium and fixed with $4 \%$ formaldehyde (Sigma-Aldrich Co.) at RT for $10 \mathrm{~min}$. Following washings with Tris-buffered saline (TBS; $50 \mathrm{mM}$ Tris, $130 \mathrm{mM} \mathrm{NaCl}$, pH 7.4), the wells were blocked with $2 \% \mathrm{BSA}$ in TBS at RT for $2 \mathrm{~h}$. Then, the cells were incubated with different concentrations of rPvTRAP antigen (starting at $60-0.1 \mu \mathrm{g} / \mathrm{ml}$ ) in TBS at $37^{\circ} \mathrm{C}$ for an hour. Wells with only BSA and HepG2 cells served as controls. Control wells of unrelated recombinant protein were also incubated with similar dilutions of a recombinant $P$. vivax Duffy-binding protein (rPvDBP). Unbound proteins were removed by washing with TBS three times for $10 \mathrm{~min}$, and incubated with polyclonal antibodies raised in mice against purified rPvTRAP or PvDBP (1:200) at RT for $90 \mathrm{~min}$. The bound antibodies to rPvTRAP and PvDBP were detected by goat anti-mouse IgG-HRP conjugate (1:25,000; SigmaAldrich Co.) at RT for $60 \mathrm{~min}$. The binding of rPvTRAP and rPvDBP (an unrelated recombinant protein as control) to HepG2 cells was measured at $490 \mathrm{~nm}$ using a microplate reader (BioTek). Absorbance at $490 \mathrm{~nm}$ was plotted against different concentrations of the examined antigens. 


\section{Indirect immunofluorescence antibody test (IFAT)}

IFAT was performed to test the ability of the anti-rPvTRAP sera of the immunized mice to recognize the native form of the rPvTRAP antigen on the sporozoite surface as well as to determine the similarity of epitopes in recombinant forms to corresponding native proteins. In this study, $P$. vivax sporozoites were obtained from Anopheles cracens that had fed on blood collected from $P$. vivax-infected patients attending malaria clinics at the Shoklo Malaria Research Unit (Mae Sot, Thailand), following a standardized procedure [55]. Multi-spot slides were coated with $20 \mu \mathrm{l} /$ well sporozoites (around 500-1000) and allowed to dry, and then fixed in a cold acetone for $10 \mathrm{~min}$. The slides were blocked with $20 \mu \mathrm{l}$ PBS containing 2.5\% BSA (Roche, Basel, Switzerland) in a dark-humidified chamber at RT for $30 \mathrm{~min}$. After the slides were washed three times with PBS (pH 7.4), the serial dilution of anti-rPvTRAP polyclonal mice sera (1:50-1:25,600) in PBS were added to the duplicated wells and incubated in a wet chamber for $60 \mathrm{~min}$. After washing three times with PBS (pH 7.4), each well was covered with $20 \mu$ fluorescein isothiocyanate (FITC)-labelled rabbit anti-mouse IgG (1:40) and Evans blue (1:100) and then left in a wet chamber for 45 min. Again, after washing three times with PBS, coverslips were placed on each slide and examined under a fluorescence microscope (Nikon E200, Tokyo, Japan) with an oil immersion objective $(100 \times)$. The serum samples obtained from the normal mice and control groups were used as negative controls.

\section{Enzyme-linked immunosorbent assay (ELISA)}

Immunized mouse sera were evaluated for anti-rPvTRAPspecific antibodies by ELISA as described previously [56] with some modifications. Briefly, rPvTRAP protein $(50 \mathrm{ng} /$ well) was diluted in a coating buffer (0.06 $\mathrm{M} \mathrm{Na}_{2} \mathrm{CO}_{3} /$ $\mathrm{NaHCO}_{3}, \mathrm{pH}$ 9.6), coated in Maxisorp flat-bottom 96-well ELISA plates (Jet Biofil) and kept at $4{ }^{\circ} \mathrm{C}$ overnight. After incubation, the plates were washed three times with PBS-T and then were blocked with $1 \times$ PBS- $1 \%$ BSA at RT for $2 \mathrm{~h}$. After washing, the sera samples were incubated in duplicate wells with 1:200 diluted sera in PBS-T in 0.5\% BSA for $90 \mathrm{~min}$. Then, the plates were incubated with 1:25,000 dilutions of goat anti-mouse IgG antibodies conjugated with HRP (Sigma-Aldrich Co.) at RT for $60 \mathrm{~min}$. Bound antibodies were visualized after adding the OPD (Sigma-Aldrich Co.) as the substrate. After 10-20 min, the reaction was stopped with $2 \mathrm{~N} \mathrm{H}_{2} \mathrm{SO}_{4}$, and $\mathrm{OD}_{490 \mathrm{~nm}}$ was read using an ELISA microplate reader (Biotek). The ELISA cutoffs were obtained from the average of the pre-immune mouse sera ( $n=20$, normal mouse sera) plus three standard deviation (SD). Moreover, for the measurement of the specific IgG subclasses response to rPvTRAP, the test was performed as described above, except for the secondary antibodies that were specific to mouse IgG1, IgG2b, IgG2c, and IgG3 antibodies (Sigma-Aldrich Co.) diluted 1:1000 and incubated at RT for $1 \mathrm{~h}$. After washing, for the detection of IgG subclasses, the plates were incubated with 1:10,000 dilution of anti-goat IgG-HRP (Sigma-Aldrich Co.) and then developed by enzyme-specific substrate, as mentioned above.

\section{Anti-rPvTRAP antibody titration and avidity}

In this study, antibody titration was performed to evaluate the end-point titers of anti-rPvTRAP raised in mice in ELISA. Sera from individual immunized mice in each group were pooled and used in serial dilutions (1:200-1:409,600), and the titration end-point of specific anti-rPvTRAP antibodies for $\operatorname{IgG}, \operatorname{IgG} 2 \mathrm{~b}$, and $\operatorname{IgG} 2 \mathrm{c}$ was determined as the last dilution of serum that had an $\mathrm{OD}_{490 \mathrm{~nm}}$ above the cutoff.

In addition, the avidity of anti-rPvTRAP IgG and its subclasses (IgG2b and IgG2c) were estimated using ELISA, as described by Hedman et al. [57] with minor modifications. Briefly, two Maxisorb plates (Jet Biofil) were coated with rPvTRAP protein (50 ng/well), and after washing step, the sera (1:200) were incubated at RT for $1 \mathrm{~h}$. In washing step, one of the plates was washed three times with PBS-T, and the other plate was washed three times with dissociation buffer containing PBS-T-urea (8 M) with vigorous shaking. Next, both plates were washed once with additional wash with PBS-T buffer. Finally, incubation with secondary antibody, washing steps, and development of enzyme reaction were performed as mentioned above for ELISA. The avidity index (AI) was calculated by the ratio of OD value of ureatreated samples by that of non-treated samples multiplied by 100 . AI values $\leq 30 \%$, between 30 and $50 \%$, and $>50 \%$ were considered as low-, intermediate-, and high-avidity anti-rPvTRAP-specific antibodies, respectively.

\section{Lymphocyte proliferation assay}

3 (4,5-Dimethylthiazol-2-yl)-2,5-diphenyltetrazolium bromide; thiazolyl-blue (MTT) dye assay was used for the mice lymphocyte proliferation. In brief, on days 42 and 180 of the first immunization, three mice from each group were sacrificed by cervical dislocation under sterile conditions. Then, the spleens were removed, and singlecell suspensions were prepared in RPMI 1640 medium (Gibco, Invitrogen, Scotland, UK). Red blood cells were eliminated with ammonium chloride-potassium lysis buffer ( $\mathrm{pH} 7.2$ ), and the cells were then washed twice and resuspended in RPMI 1640 medium, containing 5\% fetal bovine serum, $2.3 \times 10^{-2} \mathrm{mM} 2$-mercaptoethanol (2-ME), penicillin-streptomycin (100 U-100 $\mu \mathrm{g} / \mathrm{ml})$, and $10 \mathrm{mM}$ HEPES (Sigma-Aldrich Co.). The cell viability was performed by Trypan blue dye exclusion. In the next step, the 
splenocytes $\left(2.5 \times 10^{5}\right.$ cell/well $)$ were cultured in a flatbottom 96-well tissue culture plate (Orange Scientific, EU, Belgium) in triplicates in the presence of rPvTRAP $(5 \mu \mathrm{g} / \mathrm{ml})$, concanavalin A (ConA, $5 \mu \mathrm{g} / \mathrm{ml}$, as the positive control), and medium alone (as the negative control). In addition, the cultivation of splenocytes from non-immunized mice in the presence of rPvTRAP $(5 \mu \mathrm{g} / \mathrm{ml})$ was used as a negative control. All plates were incubated in a humidified atmosphere in a $5 \% \mathrm{CO}_{2}$ incubator at $37{ }^{\circ} \mathrm{C}$ for 2 days. Subsequently, the supernatants were removed, and cell proliferation was measured by the MTT assay. In this assay, $100 \mu \mathrm{l}$ MTT $(0.5 \mathrm{mg} / \mathrm{ml}$, Sigma-Aldrich Co.) was added to each well, and the plates were further incubated at $37{ }^{\circ} \mathrm{C}$ for $4 \mathrm{~h}$. Afterwards, the supernatant was removed, and the formazan crystals were dissolved in $0.04 \mathrm{~N} \mathrm{HCl}$ in absolute isopropanol, and the OD was measured at $550 \mathrm{~nm}$.

\section{Extracellular cytokine assays}

In supernatants of stimulated splenocytes of immunized mice with rPvTRAP, released cytokines were analyzed using murine cytokine immunoassay kits (R\&D system, Minneapolis, USA). After optimization at 24, 48, and $120 \mathrm{~h}$, the supernatants of splenocyte cultures, stimulated with target antigen, were collected to measure IL-4, IL-10, and IFN- $\gamma$ productions by ELISA, respectively. For the quantitative estimation of IL-4, IL-10, and IFN- $\gamma$, the frozen samples were taken out and analyzed using the standard curve, which was obtained with known concentrations of recombinant cytokines. Cytokine release profiles in immunized mice with rPvTRAP were analyzed using murine cytokine immunoassay kits (R\&D system). At days 38 and 180, three mice from the treated and control groups were sacrificed, and the spleens were isolated. After that, a single-cell suspension of splenocytes was seeded in complete RPMI 1640 medium, supplemented with 5\% FCS, $2 \mathrm{mM}$ glutamine, $2.3 \times 10^{-2} \mathrm{mM} 2$-ME, $10 \mathrm{mM}$ HEPES, and $100 \mathrm{U}-100 \mu \mathrm{g} / \mathrm{ml}$ of penicillin-streptomycin. The cells were incubated in flatbottomed, 96-well microtitre plates (Jet Biofil) at a density of $2.5 \times 10^{5}$ cells/well in the presence of $\operatorname{rPvTRAP}(5 \mu \mathrm{g} / \mathrm{ml})$. The positive and negative controls were Con A $(5 \mu \mathrm{g} / \mathrm{ml})$ and medium alone, respectively. All plates were incubated in the presence of $5 \% \mathrm{CO}_{2}$ in a humidified atmosphere at $37{ }^{\circ} \mathrm{C}$, and culture supernatants were collected after $24 \mathrm{~h}$ for IL-4, $48 \mathrm{~h}$ for IL-10, and $120 \mathrm{~h}$ for IFN- $\gamma$. The concentration of cytokines was calculated based on the standard curves performed in parallel with known concentrations of recombinant mouse IL-4, IL-10, and IFN- $\gamma$ cytokines for each experiment. All tests were performed in duplicate, and the mean of concentration \pm SD was recorded for each set of samples.

\section{Statistical analysis}

A database was created with IBM SPSS 21.0 for Windows (Armonk, NY: IBM Corp, USA). Comparisons between the groups for antibody levels and cellular responses were analyzed by one-way ANOVA. Independent sample $t$ test was used for comparison between two groups. Paired sample $t$ test was used to analyze the persistence of humoral and cellular immune responses in each group on days 38 and 180 . For all tests, $P<0.05$ was considered statistically significant.

\section{Results}

\section{Expression of rPvTRAP in E. coli}

The optimum condition for rPvTRAP expression was obtained in Terrific Broth (TB) medium where $0.2 \mathrm{mM}$ IPTG was added to the culture $\left(\mathrm{OD}_{600 \mathrm{~nm}} 0.6-0.8\right)$ after $4 \mathrm{~h}$. rPvTRAP was expressed in E. coli in large amounts for immunization, and after purification, the purity of rPvTRAP was confirmed by SDS-PAGE. The results showed a single band with a molecular mass of $\sim 70 \mathrm{kDa}$ [53], and Western blot analysis using anti-His antibody and $P$. vivax-infected human sera showed recognition of the rPvTRAP under both reducing and non-reducing conditions [53]. Protein migration at different sizes on SDS-PAGE, in the presence and absence of $2 \mathrm{ME}$, indicated disulfide bound formation in this recombinant protein [53]. The endotoxin concentration of the purified rPvTRAP was less than $0.1 \mathrm{EU} / \mathrm{ml}$, and hence, the injection of $200 \mu \mathrm{l}$ containing $\mathrm{PPvTRAP} /$ mouse demonstrated an acceptable amount (0.02 EU) of endotoxin into each mouse.

\section{Recognition of native TRAP on P. vivax parasites by mouse polyclonal antibodies to rPVTRAP}

Anti-PvTRAP antibody produced in immunized mice groups 1-6 recognized the native protein expressed on the surface of $P$. vivax sporozoite with high intensity, as indicated by the worm-like fluorescence pattern (Fig. 1a, b). This result shows the presence of common epitopes in recombinant and native forms of rPvTRAP antigen. None of the control mouse sera (groups 7-12) recognized the expressed native protein on parasite (Fig. 1c), confirming that responses were specific to rPvTRAP.

\section{Functional characterization of rPvTRAP}

To characterize the rPvTRAP, binding assay to heparan sulfate was performed. The result demonstrated that the purified rPvTRAP has a binding capacity to immobilized heparan sulfate in a concentration-dependent manner and was 

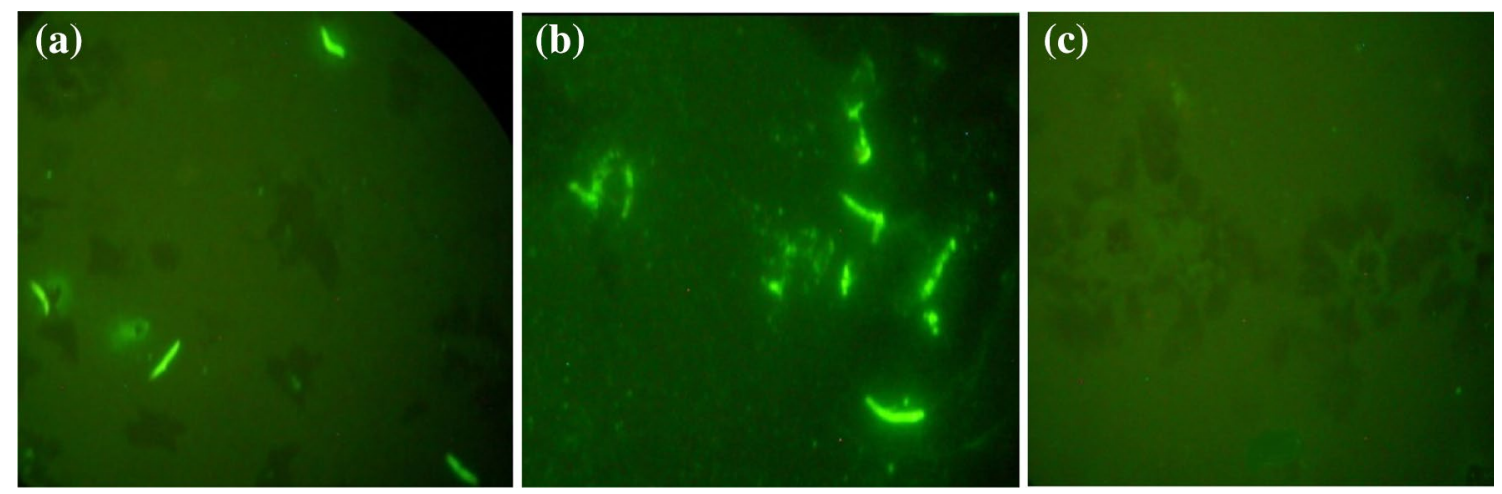

Fig. 1 Representative photographs of indirect immunofluorescence antibody test (IFAT). The pictures show the recognition of native form of PvTRAP in the surface of $P$. vivax sporozoite using polyclonal antibodies (1:100) produced in mice against rPvTRAP/NLX-
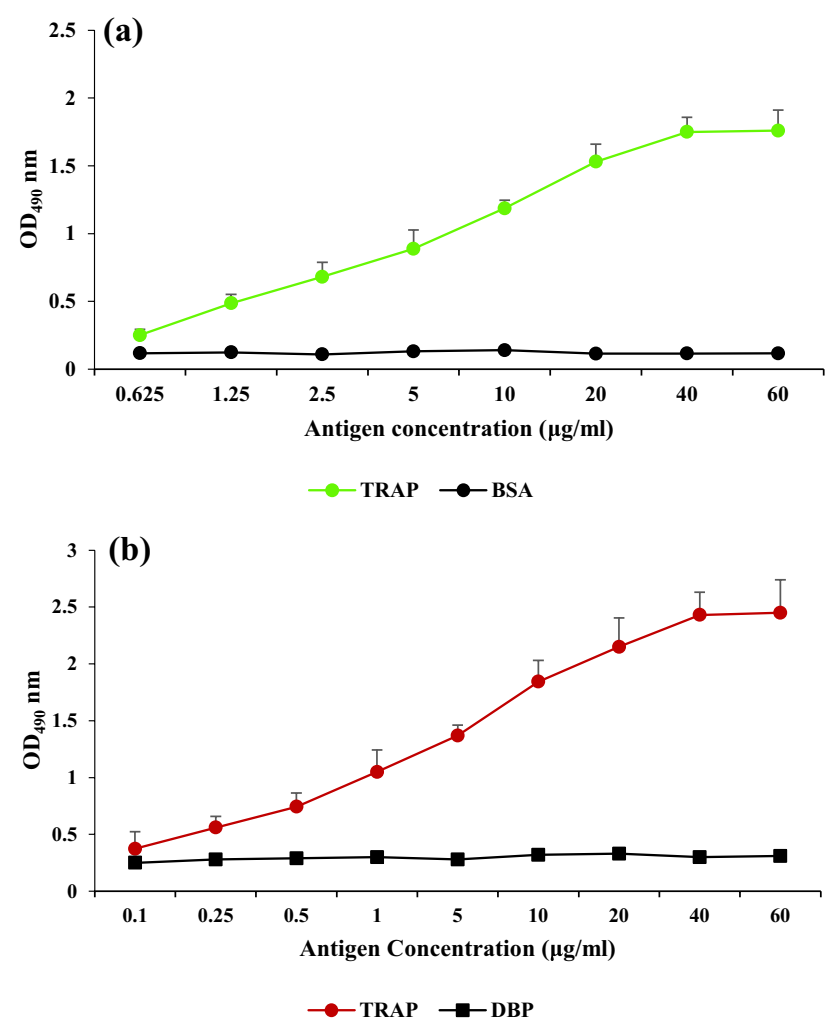

Fig. 2 Heparan sulfate and HepG2-binding assays. The results demonstrated that PvTRAP bound to immobilized heparan sulfate (a) and HepG2 cells (b) in a concentration-dependent manner. BSA and DBP were used as negative controls in heparin sulfate and HepG2-binding assay, respectively

saturable at $40 \mu \mathrm{g} / \mathrm{ml}$ concentration of the protein (Fig. 2a). Further functional characterization of rPvTRAP was performed with binding to hepatoma cell line HepG2 (IBRC C10096), and the result showed binding capacity to HepG2 liver cells in the same concentration-dependent manner
MPL-CpG (a) and rPvTRAP/CFA (b). None of the control mice sera (c), recognized the native protein on $P$. vivax parasite, ensuring that the obtained responses are specific to rPvTRAP

and was saturable at $40 \mu \mathrm{g} / \mathrm{ml}$ concentration of the protein (Fig. 2b). The lack of PvDBP (an unrelated recombinant protein, as the negative control) binding to HepG2 liver cells shows specific binding of rPvTRAP-HepG2 cells.

\section{Immunization with rPvTRAP in different adjuvant formulations and persistence of the IgG level}

To test whether different adjuvant formulations could modulate specific immune responses to PvTRAP, C57BL/6 was immunized three times with the purified rPvTRAP antigen in combination with CFA/IFA (as the reference adjuvant), as well as with NLX, MPL, and CpG individually, and with the mixture of NLX-MPL-CpG (as the adjuvant). As shown in Fig. 3a, a significant increase was detected in anti-PvTRAP IgG antibodies in all mouse groups received rPvTRAP in combination with different adjuvant formulations, on days 24 and 38 of the first immunization when compared to the first dose $(P<0.05$, paired sample $t$ test). The groups 2-6 induced higher and statistically significant specific IgG antibody in comparison the immunized mice group received $\mathrm{rPvTRAP}$ alone on day 38 of the first immunization $(P<0.05$, one-way ANOVA, Fig. 3a). However, the highest level of anti-PvTRAP IgG was identified in the group 3, which received rPvTRA/NLX-MPL-CpG (mean $\mathrm{OD}_{490 \mathrm{~nm}}$ 2.55, Table $\mathrm{S} 1$ in Online Resource 1; Fig. 3a). Furthermore, the levels of anti-PvTRAP IgG antibody among the mice immunized with rPvTRAP/ NLX-MPL-CpG were significantly different from the mouse groups that received rPvTRAP alone or in combination with NLX $(P<0.05$, one-way ANOVA, Fig. 3a), but were not significantly different from the groups that received $\mathrm{rPvTRAP/CFA/IFA,} \mathrm{PvTRAP/MPL,} \mathrm{and}$ PvTRAP/CpG alone ( $P>0.05$, one-way ANOVA, Fig. 3a). Control mice that were immunized with only adjuvant(s) did not present the detectable levels of specific antibodies 


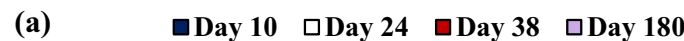

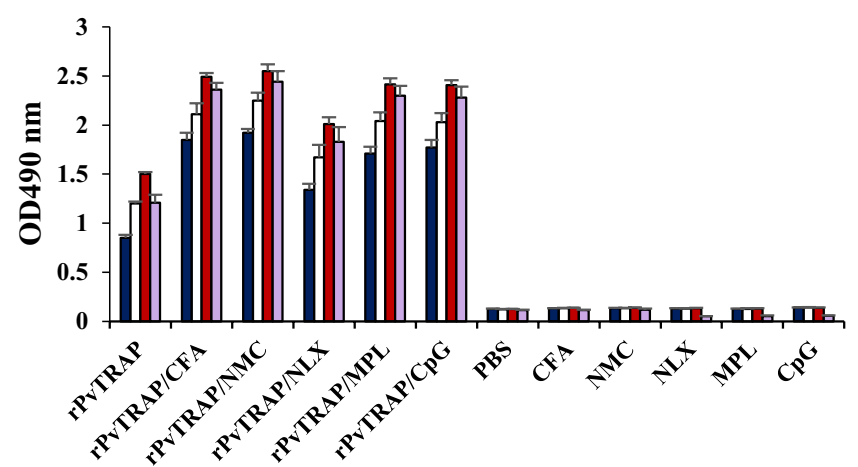

(b) $\quad$ Total IgG $\quad$ IgG1 $\square$ IgG2b $\square$ IgG2c $\square$ IgG3

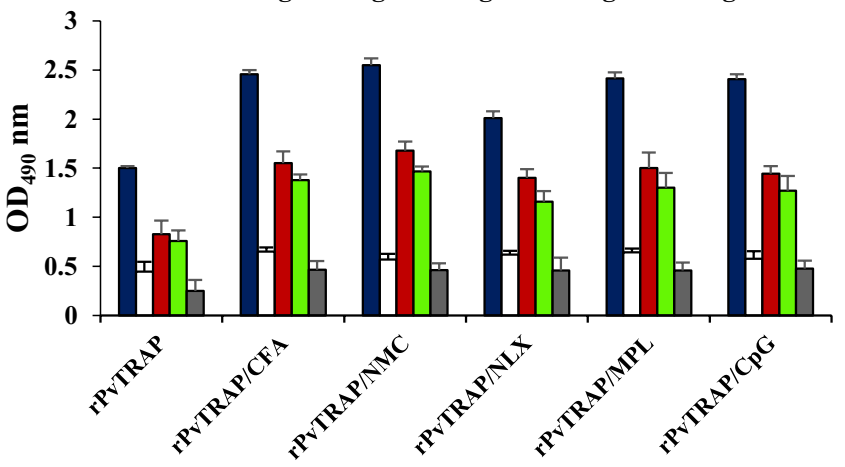

(c)

口Day 38 Day 180

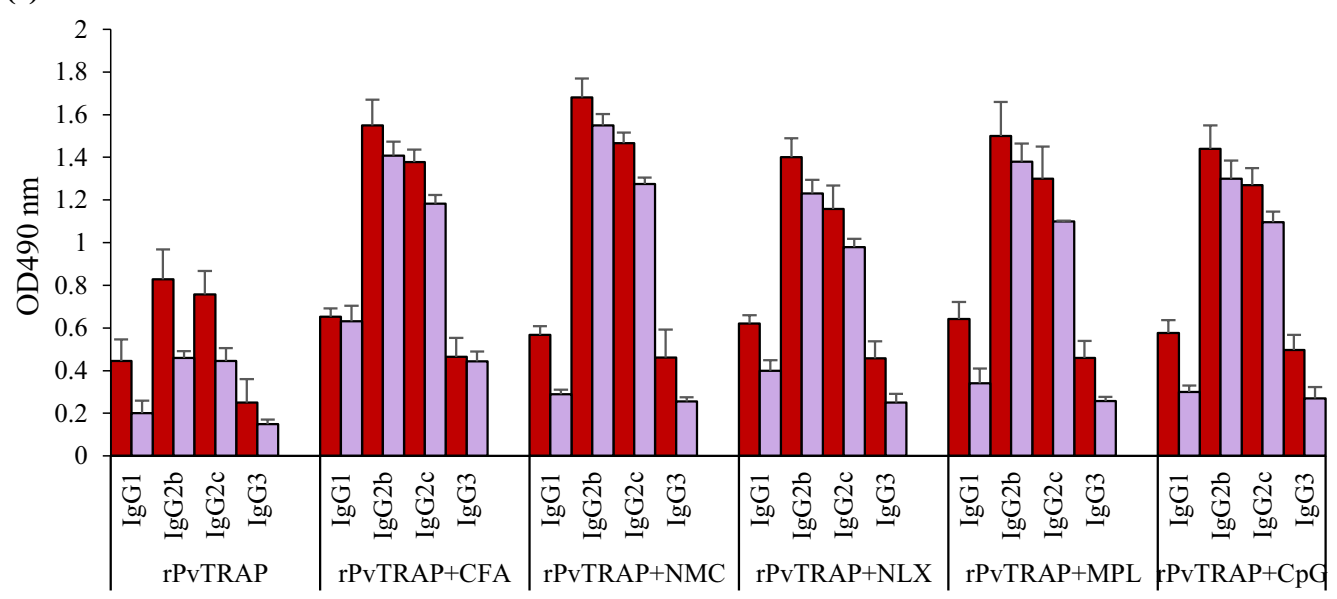

Fig. 3 Antibody responses of immunized mice to $\mathrm{PPvTRAP}$ with CFA, NLX, MPL, CpG alone and NLX-MPL-CpG in combination. a Comparison of anti-rPvTRAP IgG antibodies in immunized mice on days 10, 24 (first boost), 38 (second boost), and 180 of the first immunization. b Assessment of the IgG subclass antibodies to rPvTRAP in the sera of immunized mice on day 38 of the first immunization (after the second boost). c Evaluation of the longevity of anti-PvTRAPIgG1, IgG2b, IgG2c, and IgG3 antibodies on days 38 and 180 of the first immunization. The bars and error bars show the mean $\mathrm{OD}_{490 \mathrm{~nm}}$ and standard deviation (SD) in each mouse group $(n=15)$, respectively. The ELISA cutoffs were calculated

(Fig. 3a). Furthermore, immunoblot results using pooled sera derived from immunized mice (groups 1-6) showed that anti-PvTRAP IgG antibodies recognized the purified rPvTARP antigen (data not shown).

For determination of the longevity of the anti-PvTRAP IgG in immunized mice, sera were collected from each group on day 180 after the first immunization. The results showed no significant difference in the levels of anti-PvTRAP IgG in the mice groups receiving $\mathrm{PVTRAP} / \mathrm{NLX}-\mathrm{MPL}-\mathrm{CpG}$, / NLX, /MPL, /CpG, and /CFA/IFA on day 180 in comparison with the collected sera on day $38(P>0.05$, paired sample $t$ test, Fig. 3a). In the adjuvant groups, the highest and lowest reductions in $\mathrm{IgG}$ antibody level were observed in the group as the mean $\mathrm{OD}_{490 \mathrm{~nm}}$ of pre-immune mice sera (as the negative controls, $n=20$ ) plus $3 \mathrm{SD}$. The cutoffs for $\operatorname{IgG}$ on days $10,24,38$, and 180 were $0.147,0.149,0.152$, and 0.1 , respectively. In addition, the cutoffs for IgG1, IgG2b, IgG2c and IgG3 on day 38 were $\mathrm{OD}_{490 \mathrm{~nm}}=0.076,0.098,0.1$, and 0.043 , respectively, and were 0.068 , $0.09,0.076$, and 0.040 on day 180. NMC: NLX-MPL-CpG, Group 1: rPvTRAP, Group 2: rPvTRAP/CFA, Group 3: rPvTRAP/NLX-MPLCpG, Group 4: rPvTRAP/NLX, Group 5: rPvTRAP/MPL, Group 6: rPvTRAP/CpG, Group 7: PBS, Group 8: CFA, Group 9: NLX-MPLCpG, Group 10: NLX, Group 11: MPL, and Group 12: CpG

4 [rPvTRAP/NLX (8.95\%)] and the group 3 [rPvTRAP/ NLX-MPL-CpG (4.31\%)], respectively (Fig. 3a).

\section{IgG subclasses}

Analysis of specific IgG subtypes distribution frequencies of anti-PvTRAP antibodies in all immunized mouse groups (1-6) on day 38 of the first immunization showed that IgG2b and IgG2c were the predominant isotypes (Fig. 3b). The highest level of anti-PvTRAP IgG2b and IgG2c was identified in the group 3, which received rPvTRAP/ NLX-MPL-CpG (mean OD $490 \mathrm{~nm} 1.68$ and 1.466, respectively; Table S2 in Online Resource 2) and were significantly 
different from the mouse groups that received rPvTRAP alone (mean $\mathrm{OD}_{490 \mathrm{~nm}} 0.828$ and 0.758 , respectively) or in combination with NLX (mean $\mathrm{OD}_{490 \mathrm{~nm}} 1.40$ and 1.158 , respectively, $P<0.05$, independent sample $t$ test). However, this was not significantly different from the groups that received antigen with CFA/IFA, MPL, and CpG alone as the adjuvant $(P>0.05$, independent sample $t$ test). Moreover, there were significant differences in the mean level of anti-PvTRAP IgG1 antibody among groups $1-6(P<0.05$, one-way ANOVA, Fig. 3b). In addition, the lowest level of anti-PvTRAP IgG1 was identified in the group 3 (mean $\mathrm{OD}_{490 \mathrm{~nm}}$ 0.568; Table $\mathrm{S} 2$ in Online Resource 2; Fig. 3c) and was significantly different from the mouse group receiving rPvTRAP/CFA/IFA adjuvant $(P<0.05$, independent sample $t$ test Fig. 3c). Regarding IgG3, the groups 2-6 induced higher and statistically significant IgG3 antibody compared to the immunized mice group received rPvTRAP alone $(P<0.05$, one-way ANOVA, Fig. 3b). Furthermore, in the group 3 (rPvTRAP/NLX-MPL-CpG), the lower level of IgG3 than the group 2 (rPVTRP/CFA/IFA) was detected (Table S2 in Online Resource 2). Besides, more analysis of specific IgG subtypes showed that the group 3, which received rPvTRAP/NLX-MPL-CpG, presented the highest $\mathrm{IgG} 2 \mathrm{c} / \mathrm{IgG} 1$ (2.58) and $\mathrm{IgG} 2 \mathrm{~b} / \mathrm{IgG} 1$ (2.95) ratio when compared to all other groups, and the lowest $\mathrm{IgG} 2 \mathrm{c} / \mathrm{IgG} 1$ (1.86) and $\mathrm{IgG} 2 \mathrm{~b} / \mathrm{IgG} 1$ (2.25) ratio was observed in the group 4 (rPvTRAP/NLX), when compared to other adjuvant groups.

For determination of the longevity of the anti-PvTRAP IgG subclasses in immunized mice, sera were collected from each group on day 180 after the first immunization. No significant difference was observed in the levels of antiPvTRAP IgG2b and IgG2c in all adjuvant groups in comparison to the collected sera on day $38(P>0.05$, paired sample $t$ test, Fig. 3c). In addition, a significant difference was observed in the levels of anti-PvTRAP IgG1 and IgG3 (except rPvTRAP/CFA/IFA) in all groups in comparison with the collected sera on day $38(P<0.05$, paired sample $t$ test, Fig. 3c). The highest and lowest reductions of $\operatorname{IgG} 2 b$ and $\operatorname{IgG} 2 \mathrm{c}$ antibodies level were found in the mouse group 4 receiving rPvTRAP/NLX (12.14 and 15.54\%) and rPvTRAP/NLX-MPL-CpG (7.73 and 13.02\%), respectively (Fig. 3c). The sera samples from pre-immune mice at day 0 (negative controls) showed no detectable immune responses to rPvTRAP, ensuring that those obtained responses are specific to rPvTRAP.

\section{Antibody titer and avidity of anti-PvTRAP IgG and its subclasses in mouse groups}

The mean of serum antibody titers was evaluated on days 38 and 180 of the first immunization by ELISA. In the groups that received rPvTRAP/NLX-MPL-CpG and rPvTRAP/ CFA/IFA, the IgG end-point titer was 102,400 at both days
38 and 180 of the first immunization (Fig. 4a). However, at day 38 , in the mouse groups that received rPvTRAP/NLX, IgG end-point titer was 51,200, which reduced to 12,800 at day 180 after the first immunization. Among different vaccine formulations, NLX induced the lowest IgG2b endpoint titer at day $38(25,600)$ and $180(6400)$ of the first immunization; however, rPvTRAP/NLX-MPL-CpG as well as rPvTRAP/CFA adjuvants induced the highest end-point titer of 102,400 (at day 38) and 12,800 (at day 180) (Fig. 4b). In case of IgG2c, the mouse group that received rPvTRAP/ NLX-MPL-CpG had end-point titer of 1:102,400 at day 38 , which was higher than the mouse group immunized with antigen alone $(25,600)$ or in combination with NLX $(51,200$, Fig. $4 c)$. At day 180 , the end-point titer $(12,800)$ was detected for the groups that received antigen with NLX-MPL-CpG, CFA/IFA, MPL, and CpG (Fig. 4c).

In this study, high-avidity IgG antibodies were induced in the mouse groups immunized with different adjuvant formulations on days 38 and 180 after the primary immunization (Table 2). Regarding AI IgG2b antibody on day 38, all mouse groups had high-avidity antibodies, and on day 180 , only in the mouse group immunized with rPvTRAP/NLX, adjuvant AI was decreased to intermediate-avidity antibodies (Table 2). Moreover, high-avidity IgG2c antibodies were induced in all the mouse groups immunized with different adjuvant formulations on days 38 and 180 (Table 2). It is notable that the avidity of the mouse group receiving rPvTRAP alone was intermediate for IgG, IgG2b, and IgG2c on days 38 and 180.

\section{Cellular immune response analysis in mice immunized with the different formulations of PvTRAP adjuvants}

In this investigation, both proliferation and cytokine production in the mice immunized with rPvTRAP antigen were determined in vitro. On days 38 and 180 after the first immunization, three mice from each group were anesthetized, and splenocytes were used for the analysis. The significant proliferation of splenocytes was observed in the mouse groups 1-6 (stimulated with rPvTRAP, mean $\mathrm{OD}_{550 \mathrm{~nm}} 0.65-0.809, P<0.05$, one-way ANOVA), but not in the control mouse groups 7-12 (mean $\mathrm{OD}_{550 \mathrm{~nm}} 0.09-0.14$, $P>0.05$, one-way ANOVA). Furthermore, the proliferation of splenocytes among the immunized mice with rPvTRAP/ NLX-MPL-CpG (mean OD $_{550 \mathrm{~nm}}$ 0.775) was not significantly different from the mouse groups that received rPvTRAP with the reference adjuvant CFA (mean $\mathrm{OD}_{550 \mathrm{~nm}}$ $0.809, P>0.05$, independent sample $t$ test) but was significantly different from the mice immunized with rPvTRAP alone (mean $\mathrm{OD}_{550 \mathrm{~nm}} 0.659$ ), NLX (mean $\mathrm{OD}_{550 \mathrm{~nm}} 0.677$ ), MPL (mean $\mathrm{OD}_{550 \mathrm{~nm}} 0.597$ ), and $\mathrm{CpG}\left(\right.$ mean $\mathrm{OD}_{550 \mathrm{~nm}}$ $0.683)$ adjuvants $(P<0.05$, independent sample $t$ test $)$. In 
Day 38

IgG
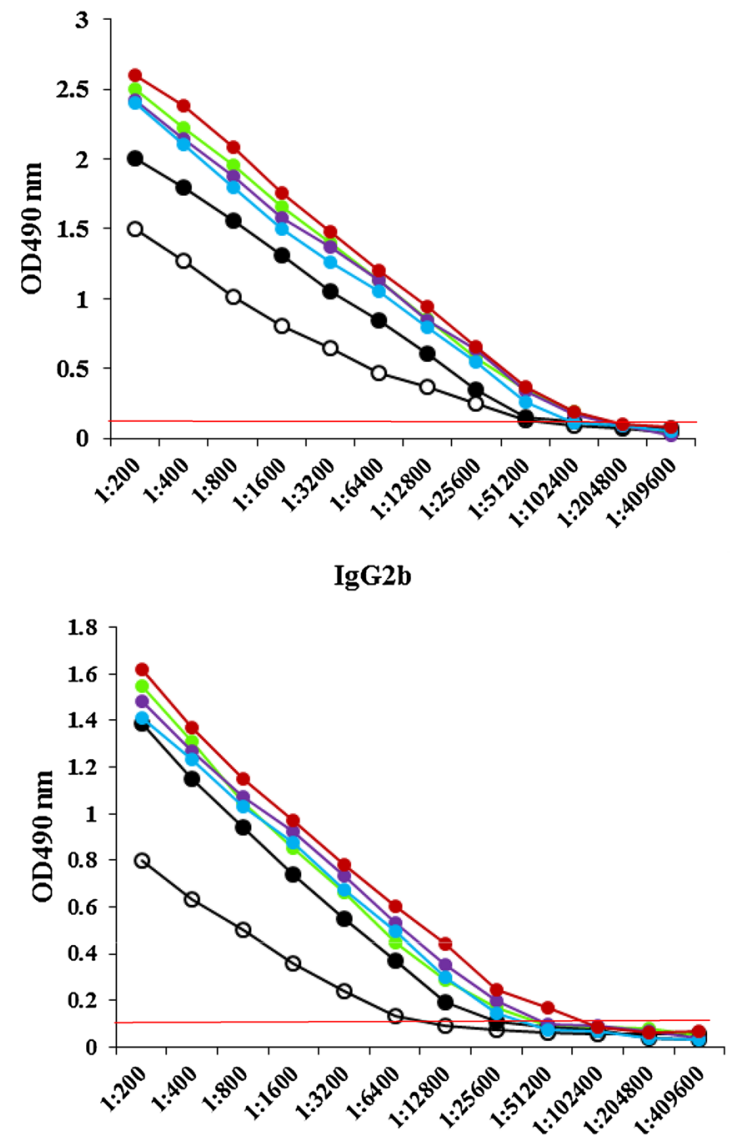

IgG2c

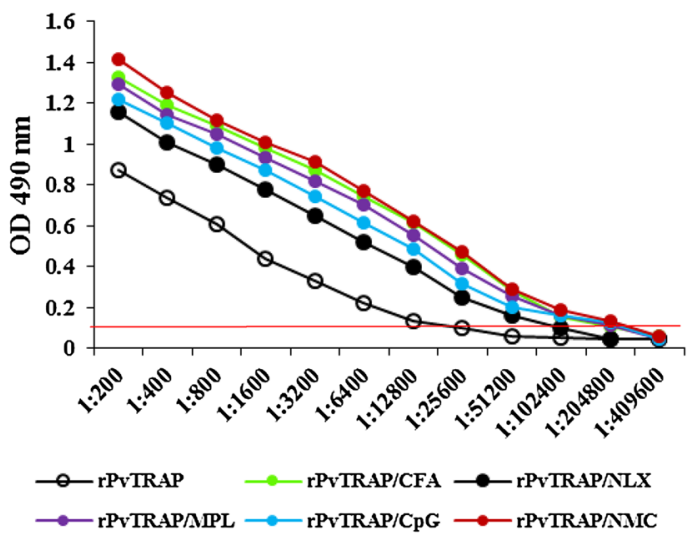

Fig. 4 Titration evaluation of anti-PvTRAP IgG, IgG2b, and $\operatorname{IgG} 2 \mathrm{c}$ antibodies on days 38 and 180 of the first immunization. No difference was observed in the end-point titres of IgG, IgG2b, and IgG2c antibodies among mouse groups immunized with either rPvTRAP/ CFA or rPvTRAP/NLX-MPL-CpG on days 38 and 180 after the first immunization. The cutoffs for $\operatorname{IgG}, \operatorname{IgG} 2 \mathrm{~b}$, and $\operatorname{IgG} 2 \mathrm{c}$ on day 38 were
Day 180

IgG
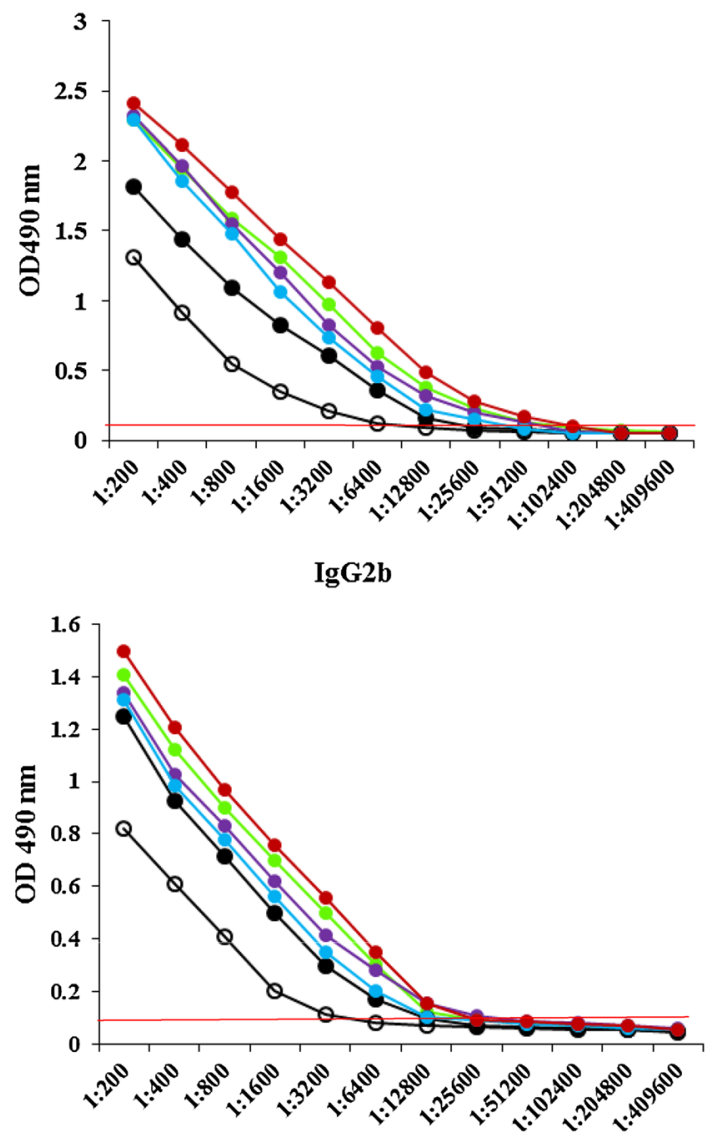

IgG2c

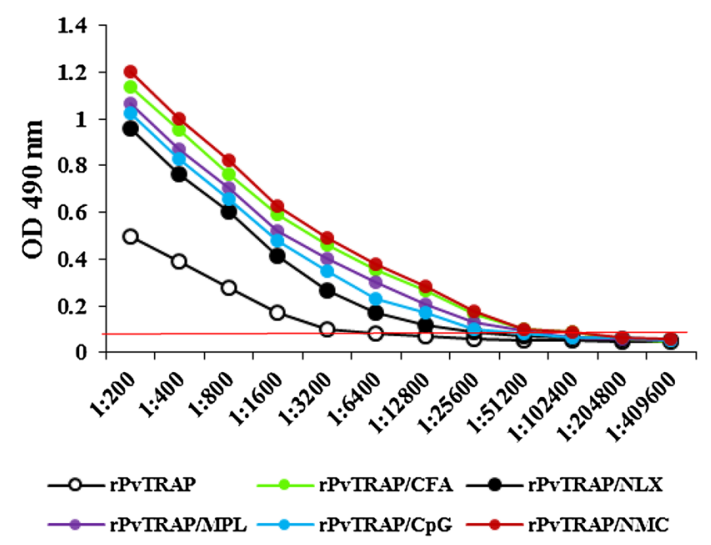

$0.152,0.098$, and 0.1 , respectively. In addition, the cutoffs for $\mathrm{IgG}$, IgG2b and IgG2c on day 180 were $0.1,0.090$, and 0.076 , respectively. Group 1: rPvTRAP; Group 2: rPvTRAP/CFA; Group 3: rPvTRAP/ NLX-MPL-CpG; Group 4: rPvTRAP/NLX; Group 5: rPvTRAP/ MPL; Group 6: rPvTRAP/CpG 
Table 2 Avidity analysis of $\operatorname{IgG}, \operatorname{IgG} 2 \mathrm{~b}$, and $\operatorname{IgG} 2 \mathrm{c}$ antibodies to rPvTRAP

\begin{tabular}{|c|c|c|c|c|c|c|}
\hline \multirow[b]{3}{*}{ Group } & \multicolumn{6}{|c|}{ Avidity index $\%(\mathrm{AI}) \pm \mathrm{SD}$} \\
\hline & \multicolumn{2}{|l|}{$\mathrm{IgG}$} & \multicolumn{2}{|l|}{$\operatorname{IgG} 2 b$} & \multicolumn{2}{|l|}{$\operatorname{IgG} 2 \mathrm{c}$} \\
\hline & Day 38 & Day 180 & Day 38 & Day 180 & Day 38 & Day 180 \\
\hline 1-rPvTRAP & $38.32 \pm 0.33$ & $31.51 \pm 0.21$ & $38.66 \pm 0.28$ & $34.49 \pm 0.20$ & $34.07 \pm 0.18$ & $33.82 \pm 0.18$ \\
\hline 2-rPvTRAP + CFA/IFA & $81.54 \pm 0.22$ & $75.41 \pm 0.25$ & $90.46 \pm 0.24$ & $80.79 \pm 0.22$ & $93.72 \pm 0.25$ & $76 \pm 0.19$ \\
\hline 3-rPvTRAP + NMC & $85.66 \pm 0.23$ & $77.79 \pm 0.22$ & $93.9 \pm 0.17$ & $82.15 \pm 0.16$ & $95.43 \pm 0.27$ & $90.47 \pm 0.16$ \\
\hline 4-rPvTRAP+NLX & $60.56 \pm 0.30$ & $51.38 \pm 0.21$ & $63.01 \pm 0.24$ & $45.74 \pm 0.16$ & $63.52 \pm 0.23$ & $50.48 \pm 0.16$ \\
\hline 5-rPvTRAP + MPL & $80.29 \pm 0.21$ & $62.52 \pm 0.17$ & $84.65 \pm 0.20$ & $76.8 \pm 0.20$ & $91.78 \pm 0.22$ & $75.09 \pm 0.18$ \\
\hline 6-rPvTRAP + CpG & $79.29 \pm 0.31$ & $54.03 \pm 0.21$ & $80.27 \pm 0.19$ & $55.04 \pm 0.20$ & $92.72 \pm 0.20$ & $62.99 \pm 0.23$ \\
\hline$P$ value & 0.0001 & 0.0001 & 0.0001 & 0.0001 & 0.0001 & 0.0001 \\
\hline
\end{tabular}

An avidity index (AI) less than $30 \%$ corresponds to low-avidity antibodies, AI between 30 and $50 \%$ to an intermediate avidity, and AI greater than $50 \%$ to high avidity. Comparisons between the groups for avidity index were analyzed by one-way ANOVA. $P<0.05$ was considered statistically significant

$S D$ standard deviation, NMC NLX-MPL-CpG, $C F A$ complete Freund's adjuvant, $I F A$ incomplete Freund's adjuvant, $N L X$ naloxone, $M P L$ monophosphoryl lipid A

addition, the stimulated splenocytes with ConA had high proliferation (mean $\mathrm{OD}_{550 \mathrm{~nm}} 0.823-1.084$ ).

The cytokine profiles of the immunized mice with rPvTRAP antigen using different adjuvants (CFA/IFA, NLX, MPL, CpG, and NLX-MPL-CpG) were determined using murine cytokine immunoassay kits (R\&D system). The results revealed the eliciting significant levels of IFN- $\gamma$ in the mouse groups received rPvTRAP antigen/adjuvants as compared to the mouse control groups (7-12) on day 38 after primary immunization $(P<0.05$, one-way ANOVA, Fig. $5 \mathrm{a})$. On day 38, the lowest level of IFN- $\gamma$ (mean $1390 \mathrm{pg} / \mathrm{ml}$ ) was produced by the mouse group that received rPvTRAP antigen without any adjuvant, while significant levels of IFN- $\gamma$ were elicited in the mouse groups immunized with rPvTRAP/NLX-MPL-CpG (mean $2492 \mathrm{pg} / \mathrm{ml})(P<0.05$, independent sample $t$ test). Besides, the level of IFN- $\gamma$ in mice receiving the antigen together with NLX-MPL-CpG was not significantly different from the mouse groups that received rPvTRAP with the reference adjuvant CFA (mean $2288 \mathrm{pg} / \mathrm{ml}$ ); however, it was significantly different from the mice immunized with NLX (mean $1615 \mathrm{pg} / \mathrm{ml}$ ), MPL (mean $2164 \mathrm{pg} / \mathrm{ml}$ ), and CpG adjuvants (mean $2029 \mathrm{pg} / \mathrm{ml}$ ) $(P<0.05$, independent sample $t$ test, Fig. 5a).

On day 180 after primary immunization, the level of IFN- $\gamma$ was significantly decreased in response to rPvTRAP in all the mouse groups $(P<0.05$, paired sample $t$ test, Fig. 5a). The most reduction in the level of IFN- $\gamma$ was observed in mice immunized with rPvTRAP alone (49.64\%), followed by NLX adjuvant $(27.80 \%)$. Despite the significant reduction of IFN- $\gamma$ in different test groups, the mouse group that received the antigen in combination with NLX-MPL-CpG adjuvant still produced the significant levels of IFN- $\gamma(2100 \mathrm{pg} / \mathrm{ml})$ in comparison to the other mouse groups, including antigen alone as well as antigen together with CFA/IFA (mean 1681 pg/ml), NLX (mean 1166 pg/ $\mathrm{ml}$ ), MPL (mean $1597 \mathrm{pg} / \mathrm{ml}$ ), and CpG adjuvants (mean $1673 \mathrm{pg} / \mathrm{ml})(P<0.05$, independent sample $t$ test, Fig. 5a).

Concerning the levels of IL-4 secretions as Th2 response, there was no significant difference in IL-4 production in different groups (1-6) as compared with that in the control groups immunized with PBS, CFA/IFA, NLX-MPL-CpG, NLX, MPL, or CpG, adjuvants $(P>0.05$, one-way ANOVA, data not shown).

Regarding IL-10 secretion as $\mathrm{T}$ regulatory (Treg) response, there was no significant difference in the mouse groups received rPvTRAP antigen/adjuvants as compared to mouse control groups (immunized with PBS, CFA/IFA, NLX-MPL-CpG, NLX, MPL, or CpG adjuvants) on days 38 (33.3-73.2) and 180 (25.2-61.1) after the primary immunization $(P>0.05$, one-way ANOVA, Fig. 5b).

\section{Discussion}

Up to now, several strategies, including subunit vaccine, have been employed to develop a novel pre-erythrocyte stage malaria vaccine, with insufficient success [58]. Vaccination with the recombinant purified protein, as an antigen, typically results in the induction of a low or the modest antibody with little or no T-cell responses, due to purification processes that impairs pathogens' essential immune stimulators (as a natural innate immune trigger) [59] as well as rapid degradation in vivo. Therefore, there is a strong demand for the use of synthetic immune enhancers capable of elevating the immunogenicity or complementing the activity of subunit vaccines in vivo. On the other hand, the selected adjuvant must be human compatible with less reactogenic profile than original Freund's 

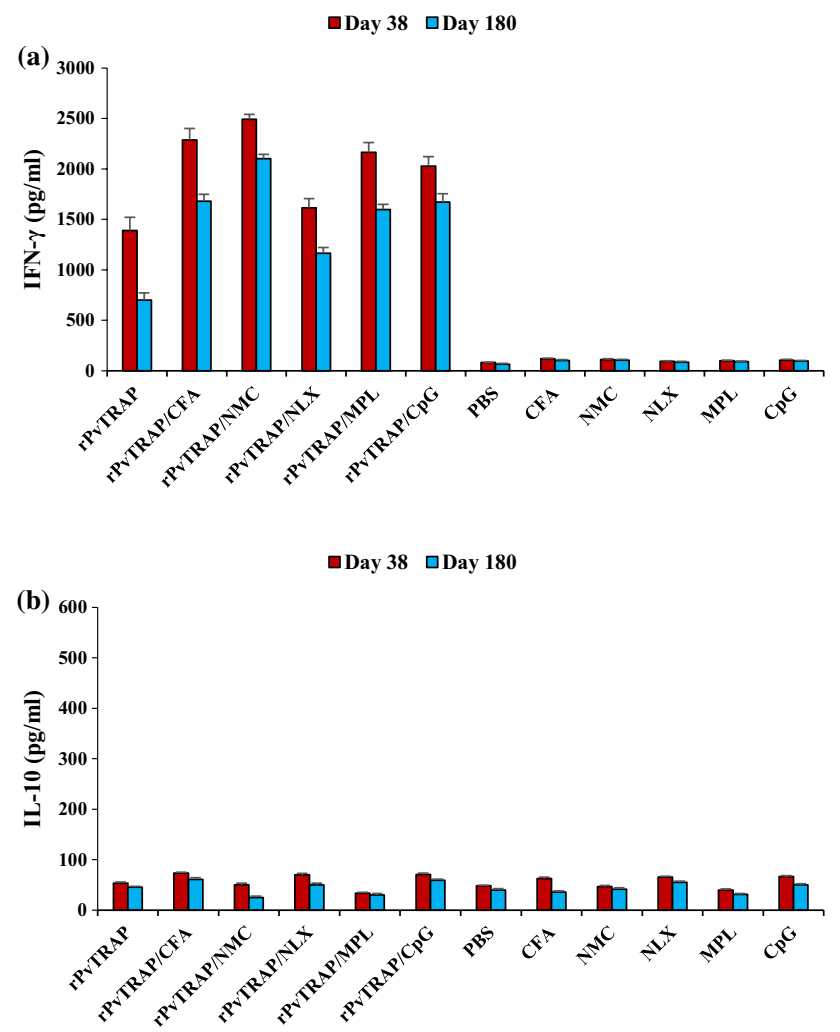

Fig. 5 Assessment of the IFN- $\gamma$ (a) and IL-10 (b) production in mice immunized with rPvTRAP alone or emulsified in different adjuvant formulations (rPvTRAP/CFA, rPvTRAP/NLX, PvTRAP/MPL, PvTRAP/CpG, and rPvTRAP/NLX-MPL-CpG) on days 38 and 180 of the first immunization. The bars show the mean concentration of elicited IFN- $\gamma$ and IL-10 from pooled lymphocytes of immunized mice $(n=3)$ in each group, and error bars indicate SD. IFN- $\gamma$ responses of ConA (as the positive control) and no antigen (as the negative control) were in the range of 1891-2097 and 12-46 pg/ml, respectively, among different examined groups. IL-10 responses of ConA (as the positive control) and no antigens (as the negative control) were in the range of $1120-1530$ and $14-18 \mathrm{pg} / \mathrm{ml}$, respectively, among different examined groups. NMC: NLX-MPL-CpG, Group 1: rPvTRAP, Group 2: rPvTRAP/CFA, Group 3: rPvTRAP/NLX-MPLCpG, Group 4: rPvTRAP/NLX, Group 5: rPvTRAP/MPL, Group 6: rPvTRAP/CpG, Group 7: PBS, Group 8: CFA, Group 9: NLX-MPLCpG, Group 10: NLX, Group 11: MPL, and Group 12: CpG

adjuvant to improve the immunogenicity of target antigen for enhancing antigen-specific adaptive immune responses [59]. In this regard, immunomodulatory molecules such as TLR4 ligand (MPL), which includes a part of the AS in the HPV vaccine, the TLR9 ligand (CpG-ODN), which is the adjuvant in the vaccine candidate for hepatitis $\mathrm{B}$ that has completed a phase 3 clinical trial [60], and NLX, as an opioid antagonist that has the ability to shift the immune response to a Th1 profile $[35,36]$ were considered. To our knowledge, so far, there is no published result regarding the evaluation of the efficacy of NLX, CPG, and MPL (as human-compatible adjuvants and potent-inducing Th1 response) as well as NLX-MPL-CpG (a novel adjuvant combination) for improving the immunogenicity of rPvTRAP in a mouse model.

It is evident that in any subunit vaccine development, the correct folding of the recombinant protein is highly important, since antibody recognition could rely on conformational epitopes; thus, in the current work, the expressed PvTRAP was evaluated for the correct folding. In our previous [53] and the current works, the results of reduced and non-reduced SDS-PAGE, Western blot analysis, IFAT, and binding assay confirmed that the bacterial rPvTRAP has been correctly folded in a native-like form. rPvTRAP has the ability to bind to heparan sulfate as well as to HepG2 cells. IFAT results also revealed that anti-rPvTRAP antibodies produced in mice recognized the native protein expressed on the surface of $P$. vivax sporozoite with high intensity that confirmed the correct structural, biological, and functional activities of rPvTRAP. However, in immunized mice, the level of IgG antibodies to rPvTRAP was significantly increased in rPvTRAP/adjuvant groups, signifying the need for an adjuvant in such vaccine formulation to augment and modulate Th1/cellular response. These results might be related to the low immunogenicity of the expressed antigen, thereby leading to the requirement for potent adjuvants.

In the present study, immunization of mice with rPvTRAP formulated with NLX, MPL, CpG, and their combination (NLX-MPL-CpG) as well as CFA/IFA (the reference adjuvant) induced a relatively high level of $\mathrm{IgG} 2 \mathrm{~b}$ and $\mathrm{IgG} 2 \mathrm{c}$ and a lower amount of $\mathrm{IgG} 1$ and $\mathrm{IgG} 3$, as compared to the mice immunized with rPvTRAP alone. This result indicates that although the rPvTRAP alone showed some degrees of the immunogenicity, its combination with the selected adjuvants could improve its immunogenicity in inducing long-lasting Th1 response, which is more favorable for the pre-erythrocyte stage of malaria vaccine. This finding is in consistent with the previous works, suggesting that NLX [31-33], MPL [61, 62], and CpG [63, 64] are able to induce Th1 immune response. Furthermore, IgG2b/IgG1 and $\mathrm{IgG} 2 \mathrm{c} / \mathrm{IgG} 1$ ratios were higher in the mice group receiving rPvTRAP/NLX-MPL-CpG, as an adjuvant, in comparison to the group that received antigen without any adjuvant. This observation provides more evidence for the ability of these adjuvants in shifting the immune response to Th1.

On day 180 of the first immunization, the percentage of reduction in IgG2b and IgG2c antibodies level in mouse group receiving $\mathrm{PPvTRAP} / \mathrm{NLX}-\mathrm{MPL}-\mathrm{CpG}$ as well as NLX, MPL, and CpG alone was relatively similar, indicating the induction of long-lasting immune responses due to the production of an antigen-specific memory B cells that persisted up to 180 days after the initial injection. This event could be also due to the presence of long-lived plasma cells that are able to produce antibodies in mice. Therefore, it can generally be concluded that for shifting immune responses to strong and specific Th1 response to PvTRAP, adjuvants 
MPL, CpG, and to a lesser extent, to NLX are suitable. Interestingly, this finding is in contrast to a previous work [56] in which NLX together with PPAMA-1 could induce mixed Th1/Th2 responses; however, this adjuvant with rPvTRAP produced more Th1 than Th2 responses, suggesting a role for the nature of antigen in the immune responses.

The immune sera from mice vaccinated with the rPvTRAP/adjuvants groups exhibited IgG antibody with the high avidity on days 38 and 180 of first immunization, which is likely due to the maturation of immune response and the selection of clonal B-cells that result in IgG avidity increases [65]. However, comparing the groups receiving antigen alone with those that received antigen together with adjuvants showed anti-TRAP IgG antibody with the intermediate avidity on days 38 and 180 after the first immunization. This result suggests that the adjuvants NLX, MPL, and $\mathrm{CpG}$ have a key role in maturation and persisting antibody responses with the high avidity over time, and these features can be considered in the rPvTRAP-based vaccine. Regarding $\operatorname{IgG} 2 b$ and $\operatorname{IgG} 2 c$, the immune sera from mice vaccinated with the rPvTRAP/CpG or MPL exhibited antibodies with a higher avidity than NLX on days 38 and 180 of the first immunization. Thus, our results indicated that the strength of antibody-eliciting responses in mice against rPvTRAP could increase the avidity and its persistence when recombinant antigen is combined with either MPL or CpG as an adjuvant. However, the role of such antibody in blocking sporozoite invasion of hepatocyte needs further investigation.

The previous studies have reported that increasing the production of IFN- $\gamma$ is one of the important mechanisms by which NLX [31, 32], MPL [66], and CpG [67] shift the immune response to Th1. In fact, CpG-ODN induces the expression of IL-12 by macrophages [68] and also indirectly activates NK cells and stimulates the IFN- $\gamma$ production [69-71]. The induction of IFN- $\gamma$ and IL-12 (which promote Th1 responses), but not IL-4 (which promotes Th2 responses), suggests that the administration of CpGODN in vivo might produce an environment favoring a Th1 immune response. Regarding MPL, two pathways (MyD88-dependent and MyD88-independent) are initiated [72]. The downstream signaling of the first pathway is inducing the expression of inflammatory mediators including IL-12, and the second one induces the expression of genes including IFN- $\gamma[73,74]$. These results are in parallel with those of the present work that indicated the administration of CPG, MPL, and NLX alone or in combination (NLX-MPL-CpG) with rPvTRAP induces significantly the higher levels of IFN- $\gamma$ production than in the control groups. However, it should be noted that the administration of NLX-MPL-CpG mixture with rPvTRAP in the immunized mice induced significantly the higher levels of IFN- $\gamma$ than those received NLX, MPL, or CpG alone. In addition, the percentage of reduction in IFN- $\gamma$ production in the mouse group receiving $\mathrm{rPvTRAP} / \mathrm{NLX}-\mathrm{MPL}-\mathrm{CpG}$ on day 180 was lower than in other immunized mouse groups, which indicates that the activation of several mechanisms in the production of IFN- $\gamma$ might generate an environment favoring a long-lasting Th1 immune response to rPvTRAP.

As there is a link between IL-4 and IgG1 antibody production, consistent with the previous works $[31,32,66$, 67], our observation showed no detectable IL-4 production after 24 and $48 \mathrm{~h}$ in different mouse groups that had a low level of detected IgG1 than IgG2c and IgG2b antibodies. Besides, it is proposed that IL-10 could promote the formation of Treg cells, leading to the inhibition of Th1 responses [75]. In this investigation, the results showed a low level of detectable IL-10 production $(<100 \mathrm{pg} / \mathrm{ml})$ in different immunized mouse groups. These results revealed that although the single-used adjuvant increased the level, avidity, and persistence of anti-TRAP antibodies, the combination of NLX-MPL-CpG could strongly stimulates Th1 cytokine than the use of the adjuvant individually. The reason behind this result could be that the differentiation of Th1 effector cells through specific cytokines as it has previously been shown that the addition of exogenous IL-12 [76] and IL-4 [77] in vitro promotes Th1 and Th2 differentiation, respectively.

In summary, this study provides new perspectives on the rational development of pre-erythrocyte malaria vaccine by screening the adjuvants that have ability to enhance subunit vaccine immunogenicity. The present result indicated that PvTRAP, as a pre-erythrocyte vaccine candidate, is immunogenic and its administration with CPG, MPL, and NLX in C57BL/6 mice induced Th1 immune responses. Furthermore, the present investigation, for the first time, revealed that the rPvTRAP delivery in the mixed formulation of those adjuvants (NLX-MPL-CpG) had more potential to increase the level, avidity, and persistence of anti-TRAP antibodies as they stimulate immune responses via different mechanisms. However, it warrants further assessment to test the blocking activity of the produced antibodies in immunized mice with different adjuvant formulations for their implication in the PvTRAP-based vaccination.

Acknowledgements We would like to thank Dr. Amir Amanzadeh for his technical advice in HepG2 culturing. This study was funded by a grant (No. 747) from Pasteur Institute of Iran to S. Zakeri and also by Ph.D. scholarship to S. Nazeri.

\section{Compliance with ethical standards}

Conflict of interest The authors declare that they have no conflict of interest.

Ethical approval All animal handling was in accordance with the ethical standards of the Laboratory Animal Science Department, Pasteur Institute of Iran. 


\section{References}

1. Cotter C, Sturrock HJ, Hsiang MS, Liu J, Phillips AA, Hwang J, Gueye CS, Fullman N, Goslin RD, Feachem RG (2013) The changing epidemiology of malaria elimination: new strategies for new challenges. Lancet 382(9895):900-911

2. Guerra CA, Howes RE, Patil AP, Gething PW, van Boeckel TP, Temperley WH, Kabaria CW, Tatem AJ, Manh BH, Elyazar IR, Baird JK, Snow RW, Hay SI (2010) The international limits and population at risk of Plasmodium vivax transmission in 2009. PLoS Negl Trop Dis 4(8):e774

3. Genton B, Dacremonte V, Rare L, Baea K, Reeder JC, Alpers MP, Muller I (2008) Plasmodium vivax and mixed infections are associated with severe malaria in children: a prospective cohort study from Papua New Guinea. PLoS Med 5(6):e127

4. Price RN, Douglas NM, Anstey NM (2009) New developments in Plasmodium vivax malaria: severe disease and the rise of chloroquine resistance. Curr Opin Infect Dis 22(5):430-435

5. Alexandre MA, Ferreira CO, Siqueira AM, Magalhães BL, Mourão MP, Lacerda MV, Alecrim Md (2010) Severe Plasmodium vivax malaria, Brazilian Amazon. Emerg Infect Dis 16(10):1611-1614

6. Feachem RGA, Phillips AA, Hwang J, Cotter Ch, Wielgosz B, Greenwood BM, Sabot O, Rodriguez MH, Abeyasinghe RR, Ghebreyesus TA, Snow RW (2010) Shrinking the malaria map: progress and prospects. Lancet 376(9752):1566-1578

7. Polley SD, McRobert L, Sutherland CJ (2004) Vaccination for vivax malaria: targeting the invaders. Trends Parasitol 20(3):99-102

8. Chitnis CE, Sharma A (2008) Targeting the Plasmodium vivax Duffy-binding protein. Trends Parasitol 24(1):29-34

9. Herrera S, Corradin G, Arevalo-Herrera M (2007) An update on the search for a Plasmodium vivax vaccine. Trends Parasitol 23(3):122-128

10. Remarque EJ, Faber BW, Kocken CH, Thomas AW (2008) Apical membrane antigen 1: a malaria vaccine candidate in review. Trends Parasitol 24(2):74-84

11. Reed SG, Bertholet S, Coler RN, Friede M (2009) New horizons in adjuvants for vaccine development. Trends Immunol 30(1):23-32

12. Reed SG, Orr MT, Fox ChB (2013) Key roles of adjuvants in modern vaccines. Nat Med 19(12):1597-1608

13. Pashine A, Valiante NM, Ulmer JB (2005) Targeting the innate immune response with improved vaccine adjuvants. Nat Med 11(4):S63-S68

14. Vogel FR (2000) Improving vaccine performance with adjuvants. Clin Infect Dis 30(3):S266-S270

15. Perez-Mazliah D, Langhorne J (2015) CD4 T-cell subsets in malaria: TH1/TH2 revisited. Front Immunol 5:671

16. Stoute JA, Slaoui M, Heppner DG, Momin P, Kester KE, Desmons P, Wellde BT, Garçon N, Krzych U, Marchand M (1997) A preliminary evaluation of a recombinant circumsporozoite protein vaccine against Plasmodium falciparum malaria. N Engl J Med 336(2):86-91

17. Stewart VA, McGrath SM, Walsh DS, Davis S, Hess AS, Ware LA, Kester KE, Cummings JF, Burge JR, Voss G, Delchambre M, Garcon N, Tang DB, Cohen JD, Heppner DG (2006) Preclinical evaluation of new adjuvant formulations to improve the immunogenicity of the malaria vaccine RTS,S/AS02A. Vaccine 24(42-43):6483-6492

18. Mettens P, Dubois PM, Demoitié MA, Bayat B, Donner MN, Bourguignon P, Stewart VA, Heppner DG Jr, Garçon N, Cohen J (2008) Improved T cell responses to Plasmodium falciparum circumsporozoite protein in mice and monkeys induced by a novel formulation of RTS,S vaccine antigen. Vaccine 26(8):1072-1082
19. Kester KE, Cummings JF, Ofori-Anyinam O, Ockenhouse CF, Krzych U, Moris P, Schwenk R, Nielsen RA, Debebe Z, Pinelis E, Juompan L, Williams J, Dowler M, Stewart VA, Wirtz RA, Dubois MC, Lievens M, Cohen J, Ballou WR, Heppner DG Jr, RTS,S Vaccine Evaluation Group (2009) Randomized, doubleblind, phase 2 a trial of falciparum malaria vaccines RTS,S/ AS01B and RTS,S/AS02A in malaria-naïve adults: safety, efficacy, and immunologic associates of protection. J Infect Dis 200(3):337-346

20. Agnandji ST, Fernandes JF, Bache EB, Ramharter M (2015) Clinical development of RTS, S/AS malaria vaccine: a systematic review of clinical phase I-III trials. Future Microbiol 10(10):1553-1578

21. Gosling R, von Seidlein L (2016) The future of the RTS,S/AS01 malaria vaccine: an alternative development plan. PLoS Med 13(4):e1001994

22. Ajua A, Lell B, Agnandji ST, Asante KP, Owusu-Agyei S, Mwangoka G, Mpina M, Salim N, Tanner M, Abdulla S, Vekemans J, Jongert E, Lievens M, Cambron P, Ockenhouse CF, Kremsner PG, Mordmüller B (2015) The effect of immunization schedule with the malaria vaccine candidate RTS,S/AS01E on protective efficacy and anti-circumsporozoite protein antibody avidity in African infants. Malar J 14:72

23. Kester KE, Gray Heppner D Jr, Moris P, Ofori-Anyinam O, Krzych U, Tornieporth N, McKinney D, Delchambre M, Ockenhouse CF, Voss G, Holland C, Beckey JP, Ballou WR, Cohen J (2014) RTS,S/TRAP Group. Sequential phase 1 and phase 2 randomized, controlled trials of the safety, immunogenicity and efficacy of combined pre-erythrocytic vaccine antigens RTS,S and TRAP formulated with AS02 Adjuvant System in healthy, malaria naïve adults. Vaccine 32(49):6683-6691

24. Schwarz TF, Spaczynski M, Schneider A, Wysocki J, Galaj A, Perona P, Poncelet S, Zahaf T, Hardt K, Descamps D, Dubin G (2009) Immunogenicity and tolerability of an HPV-6/18 AS04adjuvanted prophylactic cervical cancer vaccine in women aged 15-55 years. Vaccine 27(4):581-587

25. Toubaji A, Hill S, Terabe M, Qian J, Floyd T, Simpson RM, Berzofsky JA, Khleif SN (2007) The combination of GM-CSF and IL-2 as local adjuvant shows synergy in enhancing peptide vaccines and provides long term tumor protection. Vaccine 25(31):5882-5891

26. Sui Y, Zhu Q, Gagnon S, Dzutsev A, Terabe M, Vaccari M, Venzon D, Klinman D, Strober W, Kelsall B, Franchini G, Belyakov IM, Berzofsky JA (2010) Innate and adaptive immune correlates of vaccine and adjuvant-induced control of mucosal transmission of SIV in macaques. Proc Natl Acad Sci USA 107(21):9843-9848

27. Giannini SL, Hanon E, Moris P, Van Mechelen M, Morel S, Dessy F, Fourneau MA, Colau B, Suzich J, Losonksy G, Martin MT, Dubin G, Wettendorff MA (2006) Enhanced humoral and memory B cellular immunity using HPV16/18 L1 VLP vaccine formulated with the MPL/aluminium salt combination (AS04) compared to aluminium salt only. Vaccine 24(33-34):5937-5949

28. Wack A, Baudner BC, Hilbert AK, Manini I, Nuti S, Tavarini S, Scheffczik H, Ugozzoli M, Singh M, Kazzaz J, Montomoli E, Del Giudice G, Rappuoli R, O'Hagan DT (2008) Combination adjuvants for the induction of potent, long-lasting antibody and T-cell responses to influenza vaccine in mice. Vaccine 26(4):552-561

29. Singh M, Kazzaz J, Ugozzoli M, Baudner B, Pizza M, Giuliani M, Hawkins LD, Otten G, O'Hagan DT (2012) MF59 oil-in-water emulsion in combination with a synthetic TLR4 agonist (E6020) is a potent adjuvant for a combination Meningococcus vaccine. Hum Vaccin Immunother 8(4):486-490

30. Sacerdote P, di San Secondo VE, Sirchia G, Manfredi B, Panerai AE (1998) Endogenous opioids modulate allograft rejection time in mice: possible relation with Th1/Th2 cytokines. Clin Exp Immunol 113(3):465-469 
31. Sacerdote P, Gaspani L, Panerai AE (2000) The opioid antagonist naloxone induces a shift from type 2 to type 1 cytokine pattern in normal and skin-grafted mice. Ann N Y Acad Sci 917:755-763

32. Sacerdote P, Manfredi B, Gaspani L, Panerai AE (2000) The opioid antagonist naloxone induces a shift from type 2 to type 1 cytokine pattern in BALB/cJ mice. Blood 95(6):2031-2036

33. Jamali A, Mahdavi M, Shahabi S, Hassan ZM, Sabahi F, Javan M, Farsani MJ, Parsania M, Bamdad T (2007) Naloxone, an opioid receptor antagonist, enhances induction of protective immunity against HSV-1 infection in BALB/c mice. Microb Pathog 43(5-6):217-223

34. Jamali A, Mahdavi M, Hassan ZM, Sabahi F, Farsani MJ, Bamdad T, Soleimanjahi H, Motazakker M, Shahabi S (2009) A novel adjuvant, the general opioid antagonist naloxone, elicits a robust cellular immune response for a DNA vaccine. Int Immunol 21(3):217-225

35. Jazani NH, Parsania S, Sohrabpour M, Mazloomi E, Karimzad M, Shahabi S (2010) Naloxone and alum synergistically augment adjuvant activities of each other in a mouse vaccine model of Salmonella typhimurium infection. Immunobiology 216(6):744-751

36. Motaharinia Y, Rezaee MA, Rashidi A, Jalili A, Rezaie MJ, Shapouri R, Hossieni W, Rahmani MR (2013) Induction of protective immunity against brucellosis in mice by vaccination with a combination of naloxone, alum, and heat-killed Brucella melitensis 16 M. J Microbiol Immunol Infect 46(4):253-258

37. McCarthy L, Wetzel M, Sliker JK, Eisenstein TK, Rogers TJ (2001) Opioids, opioid receptors, and the immune response. Drug Alcohol Depend 62(2):111-123

38. O'Neill LAJ, Bowie AG (2007) The family of five: TIR domaincontaining adaptors in toll-like receptor signaling. Nat Rev Immunol 7(5):353-364

39. Casares S, Brumeanu TD, Richie TL (2010) The RTS,S malaria vaccine. Vaccine 28(31):4880-4894

40. Tielemans CL, lasak JV, Kosaetal D (2011) Immunogenicity and safety of an investigational AS02v-adjuvanted hepatitis B vaccine in patients with renal insufficiency who failed to respond or to maintain antibody levels after prior vaccination: results of two open, randomized, comparative trials. Vaccine 29(6):1159-1166

41. Cluf CW (2010) Monophosphoryl lipid a (MPL) as an adjuvant for anti-cancer vaccines: clinical results. Adv Exp Med Biol 667:111-123

42. Gillard P, Yang PC, Danilovits M, Su WJ, Cheng SL, Pehme L, Bollaerts A, Jongert E, Moris P, Ofori-Anyinam O, Demoitié MA, Castro M (2016) Safety and immunogenicity of the M72/AS01E candidate tuberculosis vaccine in adults with tuberculosis: a phase II randomised study. Tuberculosis (Edinb) 100:118-127

43. Van Braeckel E, Bourguignon P, Koutsoukos M, Clement F, Janssens M, Carletti I, Collard A, Demoitié MA, Voss G, LerouxRoels G, McNally L (2011) An adjuvanted polyprotein HIV-1 vaccine induces polyfunctional cross-reactive $\mathrm{CD} 4+\mathrm{T}$ cell responses in seronegative volunteers. Clin Infect Dis 52(4):522-531

44. Krieg AM (2002) CpG motifs in bacterial DNA and their immune effects. Annu Rev Immunol 20:709-760

45. Harandi AM, Holmgren J (2004) CpG DNA as a potent inducer of mucosal immunity: implications for immune prophylaxis and immunotherapy of mucosal infections. Curr Opin Investig Drugs $5(2): 141-145$

46. Krieg AM (2004) Antitumor applications of stimulating toll-like receptor 9 with $\mathrm{CpG}$ oligodeoxynucleotides. Curr Oncol Rep 6(2):88-95

47. Gupta GK, Agrawal DK (2010) CpG oligodeoxynucleotides as TLR9 agonists: therapeutic application in allergy and asthma. Bio Drugs 24(4):225-235

48. Vanderberg J, Nussenzweig R, Most H (1969) Protective immunity produced by the injection of $\mathrm{X}$-irradiated sporozoites of
Plasmodium berghei. V. In vitro effects of immune serum on sporozoites. Mil Med 134(10):1183-1190

49. Schwenk R, Asher LV, Chalom I, Lanar D, Sun P, White K, Keil D, Kester KE, Stoute J, Heppner DG, Krzych U (2003) Opsonization by antigen-specific antibodies as a mechanism of protective immunity induced by Plasmodium falciparum circumsporozoite protein-based vaccine. Parasite Immunol 25(1):17-25

50. Muller H, Reckmann I, Hollingdale MR, Bujard H, Robson KJ, Crisanti A (1993) Thrombospondin related adhesion protein (TRAP) of Plasmodium falciparum binds specifically to sulfated glycoconjugates and to HepG2 hepatoma cells suggesting a role for this molecule in sporozoite invasion of hepatocytes. EMBO J 12(7):2881-2889

51. Sinnis P, Sim BK (1997) Cell invasion by the vertebrate stages of Plasmodium. Trends Microbiol 5(2):52-58

52. Nazeri S, Mehrizi AA, Djadid ND, Zakeri S (2015) A comparative study on worldwide genetic diversity and population structure analysis of Plasmodium vivax thrombospondin-related adhesive protein (PvTRAP) and its implications for the vivax vaccine design. Infect Genet Evol 36:410-423

53. Nazeri S, Zakeri S, Mehrizi AA, Djadid ND (2017) Naturally acquired immune responses to thrombospondin-related adhesion protein (TRAP) of Plasmodium vivax in patients from areas of unstable malaria transmission. Acta Trop 173:45-54

54. Yadava A, Sattabongkot J, Washington MA, Ware LA, Majam V, Zheng H, Kumar S, Ockenhouse CF (2007) A novel chimeric Plasmodium vivax circumsporozoite protein induces biologically functional antibodies that recognize both VK210 and VK247 sporozoites. Infect Immun 75(3):1177-1185

55. Andolina C, Landier J, Carrara V, Chu CS, Franetich JF, Roth A, Rénia L, Roucher C, White NJ, Snounou G, Nosten F (2015) The suitability of laboratory-bred Anopheles cracens for the production of Plasmodium vivax sporozoites. Malar J 14:312

56. Someabozorg MA, Mirkazemi S, Mehrizi AA, Shokri F, Djadid ND, Zakeri S (2015) Administration of naloxone in combination with recombinant Plasmodium vivax AMA-1 in BALB/c mice induces mixed Th1/Th2 immune responses. Parasite Immunol 37(10):521-532

57. Hedman K, Lappalainen M, Seppaia I, Makela O (1989) Recent primary toxoplasma infection indicated by a low avidity of specific IgG. J Infect Dis 159(4):736-740

58. Herrington DA, Clyde DF, Losonsky G, Cortesia M, Murphy JR, Davis J, Baqar S, Felix AM, Heimer EP, Gillessen D (1987) Safety and immunogenicity in man of a synthetic peptide malaria vaccine against Plasmodium falciparum sporozoites. Nature 328(6127):257-259

59. Garcon N, Leroux-Roels G, Cheng WF (2011) Vaccine adjuvants. Perspect Vaccinol 1(1):89-113

60. Eng NF, Bhardwaj N, Mulligan R, Diaz-Mitoma F (2013) The potential of 1018 ISS adjuvant in hepatitis B vaccines. Hum Vaccin Immunother 9(8):1661-1672

61. Fransen F, Boog CJ, van Putten JP, van der Ley P (2007) Agonists of toll-like receptors $3,4,7$, and 9 are candidates for use as adjuvants in an outer membrane vaccine against Neisseria meningitidis serogroup. Infect Immun 75(12):5939-5946

62. Rhee EG, Kelley RP, Agarwal I, Lynch DM, La Porte A, Simmons NL, Clark SL, Barouch DH (2010) TLR4 ligands augment antigen-specific CD8+ T lymphocyte responses elicited by a viral vaccine vector. J Virol 84(19):10413-10419

63. Sugai T, Mori M, Nakazawa M, Ichino M, Naruto T, Kobayashi N, Kobayashi Y, Minami M, Yokota S (2005) A CpG-containing oligodeoxynucleotide as an efficient adjuvant counterbalancing the Th1/Th2 immune response in diphtheria-tetanus-pertussis vaccine. Vaccine 23(46-47):5450-5456

64. Fogg CN, Americo JL, Lustig S, Huggins JW, Smith SK, Damon I, Resch W, Earl PL, Klinman DM, Moss B (2007) 
Adjuvant-enhanced antibody responses to recombinant proteins correlates with protection of mice and monkeys to orthopoxvirus challenges. Vaccine 25(15):2787-2799

65. Thomas HI, Wilson S, O'Toole CM, Lister CM, Saeed AM, Watkins P, Morgan-Capner P (1996) Differential maturation of avidity of IgG antibodies to gp41, p24 and p17 following infection with HIV-1. Clin Exp Immunol 103(2):185-191

66. Varikuti S, Oghumu S, Natarajan G, Kimble J, Sperling RH, Moretti E, Kaplan MH, Satoskar AR (2016) STAT4 is required for the generation of Th1 and Th2, but not Th17 immune responses during monophosphoryl lipid A adjuvant activity. Int Immunol 28(11):565-570

67. Krug A, Rothenfusser S, Hornung V, Jahrsdörfer B, Blackwell S, Ballas ZK, Endres S, Krieg AM, Hartmann G (2001) Identification of $\mathrm{CpG}$ oligonucleotide sequences with high induction of IFN- $\alpha / \beta$ in plasmacytoid dendritic cells. Eur J Immunol 31(7):2154-2163

68. Chace JH, Hooker NA, Midlenstein KL, Krieg AM, Cowdery JS (1997) Bacterial DNA-induced NK cell IFN-g production is dependent on macrophage secretion of IL-12. Clin Immunol Immunopathol 84(2):185-193

69. Yamamoto S, Yamamoto T, Shimada S, Kuramoto E, Yano O, Kataoka T, Tokunaga T (1992) DNA from bacteria, but not from vertebrates, induces interferons, activates natural killer cells and inhibits tumor growth. Microbiol Immunol 36(9):983-997
70. Ballas ZK, Rasmussen WL, Krieg AM (1996) Induction of NK activity in murine and human cells by $\mathrm{CpG}$ motifs in oligodeoxynucleotides and bacterial DNA. J Immunol 157(5):1840-1845

71. Cowdery JS, Chace JH, Yi AK, Krieg AM (1996) Bacterial DNA induces NK cells to produce IFN-g in vivo and increases the toxicity of lipopolysaccharides. J Immunol 156(12):4570-4575

72. Shi Y, Felder MA, Sondel PM, Rakhmilevich AL (2015) Synergy of anti-CD40, CpG and MPL in activation of mouse macrophages. Mol Immunol 66(2):208-215

73. Vilaysane A, Muruve DA (2009) The innate immune response to DNA. Semin Immunol 21(4):208-214

74. Kumagai Y, Takeuchi O, Aaira S (2008) TLR9 as a key receptor for the recognition of DNA. Adv Drug Deliv Rev 60(7):795-804

75. Cooper CL, Ahluwalia NK, Efler SM, Vollmer J, Krieg AM, Davis HL (2008) Immunostimulatory effects of three classes of CpG oligodeoxynucleotides on PBMC from HCV chronic carriers. J Immune Based Ther Vaccines 6:3

76. Amsen D, Spilianakis CG, Flavell RA (2009) How are T(H)1 and $\mathrm{T}(\mathrm{H}) 2$ effector cells made? Curr Opin Immunol 21(2):153-160

77. Ansel KM, Djuretic I, Tanasa B, Rao A (2006) Regulation of Th2 differentiation and I14 locus accessibility. Annu Rev Immunol 24:607-656 